\title{
Axiomatic Characterization of Game-Theoretic Centrality
}

\author{
Oskar Skibski \\ Institute of Informatics, University of Warsaw \\ 02-097 Warsaw, Poland
}

Tomasz P. Michalak

Institute of Informatics, University of Warsaw

02-097 Warsaw, Poland

Talal Rahwan

Department of Computer Science

Masdar Institute, Khalifa University

Abu Dhabi, United Arab Emirates
OSKAR.SKIBSKI@MIMUW.EDU.PL

TOMASZ.MICHALAK@MIMUW.EDU.PL

TALAL.RAHWAN@KU.AC.AE

\begin{abstract}
One of the fundamental research challenges in network science is centrality analysis, i.e., identifying the nodes that play the most important roles in the network. In this article, we focus on the game-theoretic approach to centrality analysis. While various centrality indices have been recently proposed based on this approach, it is still unknown how general is the game-theoretic approach to centrality and what distinguishes some game-theoretic centralities from others. In this article, we attempt to answer this question by providing the first axiomatic characterization of game-theoretic centralities. Specifically, we show that every possible centrality measure can be obtained following the game-theoretic approach. Furthermore, we study three natural classes of game-theoretic centrality, and prove that they can be characterized by certain intuitive properties pertaining to the well-known notion of Fairness due to Myerson.
\end{abstract}

\section{Introduction}

Centrality analysis is one of the fundamental research problems in graph theory and network science. It involves identifying the nodes that play the most important role in the network (Brandes \& Erlebach, 2005). On top of the already classic centrality indices (or, simply, centralities) such as degree, closeness, betweenness, eigenvector, Katz, and PageRank centralities, various new concepts have been recently proposed in the literature (Koschützki, Lehmann, Peeters, Richter, Tenfelde-Podehl, \& Zlotowski, 2005).

One family of centralities that has recently attracted growing attention is based on Cooperative, or "Coalitional", Game Theory (Gomez, González-Arangüena, Manuel, Owen, del Pozo, \& Tejada, 2003; del Pozo, Manuel, González-Arangüena, \& Owen, 2011; Michalak, Aadithya, Szczepański, Ravindran, \& Jennings, 2013; Szczepański, Michalak, \& Rahwan, 2016). Here, the key idea is to analyse the topology of the network using the combinatorial structure of a coalitional game. More in detail, in the first step, one has to define a function, called a representation function, which evaluates the centrality of each subset of nodes. Next, having evaluated all the subsets, one can evaluate the role of individual nodes by applying payoff division schemes from cooperative game theory - such as the Shapley value 
or the Banzhaf power index (Chalkiadakis, Elkind, \& Wooldridge, 2011) -which measures the contribution of individual entities to the performance of the system as a whole.

Centrality indices built in such a way are called game-theoretic centralities. On the one hand, they extend conventional centrality indices which solely focus on the performance of individual nodes and neglect the performance of groups of nodes. On the other hand, gametheoretic centralities take into account the role of a node not only in the whole network, but also smaller parts formed by subset of nodes. In result, various centralities obtained using the game-theoretic approach have been shown to outperform classic centralities in a number of real-life applications, including information diffusion (Suri \& Narahari, 2008), terroristnetwork analysis (Lindelauf, Hamers, \& Husslage, 2013; Michalak, Rahwan, Skibski, \& Wooldridge, 2015), and genes and brain networks (Kötter, Reid, Krumnack, Wanke, \& Sporns, 2007; Moretti, Fragnelli, Patrone, \& Bonassi, 2010).

One example is the work by Bianzino, Chaudet, Rossi, Rougier, and Moretti (2011) on reducing the energy consumption in communication networks such as backbone IP-networks. In particular, to enable entering the energy-saving sleep mode by some of the devices, the authors studied a technique called resource consolidation that relies on concentrating the workload of an infrastructure on a reduced set of devices. To decide which devices should enter the sleep mode, the authors proposed using a game-theoretic centrality. First, for each group of devices, the subgraph induced by them is analysed to compute the amount of traffic that this group can effectively transport. Then, the Shapley value is used to assess the importance of each device. Based on the numerical results obtained over a real network topology and traffic matrix, the authors showed that using the new method leads to the improvement of the network quality of service.

Despite the recent attention that game-theoretic centralities have received in the literature, their theoretical foundations and properties are not entirely understood to date. Unfortunately, this problem, termed "theory gap", concerns not only novel centrality indices but also the classic ones, as well as many other concepts in social network analysis (Schoch \& Brandes, 2015). A few attempts to bridge this gap include the works by Sabidussi (1966), Nieminen (1974), and Boldi and Vigna (2014), who provided axiomatic characterizations of some of the classic centrality indices (see the next section for more details).

Meager axiomatic foundations are especially striking in the case of game-theoretic centralities. This is because the axiomatizations of the payoff division schemes upon which the game-theoretic indices are built have been extensively studied in the literature. Perhaps the most widely-acclaimed such division scheme is the Shapley value (Shapley, 1953) which has been axiomatized in a variety of ways (Winter, 2002). For example, one of the celebrated axiomatizations of the Shapley value is due to Myerson (1980) who showed that this payoff division scheme is uniquely determined only by two basic axioms: Efficiency and Balanced Contributions. The former axiom simply requires that the sum of all the players' payoffs is equal the total reward from cooperation, i.e., equal to the value of the grand coalition (which is the coalition containing all the players in the game). The latter axiom requires that the removal of player $i$ from the game affects the payoff of player $j$ in the same way that the removal of player $j$ affects the payoff of player $i$.

Unfortunately, despite the many axiomatizations that have been proposed for various game-theoretic payoff division schemes to date, it turns out that developing axiomatizations for game-theoretic centralities is far from straightforward. This is mainly because various 
axioms that are desirable in the context of cooperative games lose their attractiveness in the context of network centrality. For instance, it is unclear why Efficiency should be met by some game-theoretic centralities. ${ }^{1}$ Luckily, however, certain axioms from cooperative games can be directly used in the centrality context. One such axiom that will play a crucial role in this article is Fairness. This axiom was introduced by Myerson (1977) for coalitional games in which cooperation between players is restricted by an underlying graph. In essence, Fairness requires that each edge in the graph equally affects the payoff of both adjacent players. This directly translates to the centrality context, by requiring that each edge equally affects the centrality of both adjacent nodes. Indeed, such a direct translation of Fairness constitutes one of the axioms used by Skibski, Rahwan, Michalak, and Yokoo (2016), who proposed the first axiomatic characterization of a particular instantiation of game-theoretic centrality.

Nevertheless, little is known to date about the axiomatic underpinnings of the gametheoretic indices in general. Specifically, we still do not know the answers to the following two fundamental questions:

\section{How general is the game-theoretic approach to centrality?}

\section{What distinguishes some game-theoretic centrality indices from others?}

In this article, we present the first attempt to tackle both questions. In particular, we answer the first question by showing that every non game-theoretic centrality can also be obtained using some game-theoretic centrality. This result is a testimony to the versatility of the game-theoretic approach to centrality analysis. Indeed, each game-theoretic centrality is a combination of a representation function and a payoff division scheme, and both of these components can be chosen arbitrarily to suit the application at hand. We then extend this result by showing that each payoff division scheme taken from a large class, called the positive semivalues, can be used to obtain every possible centrality.

To answer the second question, we distinguish three natural classes from the set of all game-theoretic centralities, and lay axiomatic foundations for each of them. The classes differ in terms of the representation function and how it assigns values to subsets of nodes. In particular, since the representation function in its general form is meant to express any setting, it assigns a value to any subset of nodes arbitrarily, i.e., based on the topology of the entire graph and not only on the subset itself. To put it differently, in the general case, the value of the same subset of nodes can be arbitrarily different in one graph compared to another graph, no matter how small the difference between the two graphs is.

However, various instantiations of game-theoretic centrality that were studied in the literature assume some kind of dependency between the values of the same subset of nodes in different graphs (see the next section for a number of examples). Driven by this observation, we define the following three classes of representation functions and their corresponding game-theoretic centrality indices:

1. One of the centralities for which Efficiency does not seem to be a suitable axiom is the game-theoretic betweenness centrality as defined in the work by Szczepański et al. (2016), where the value of the grand coalition happens to be 0 . 


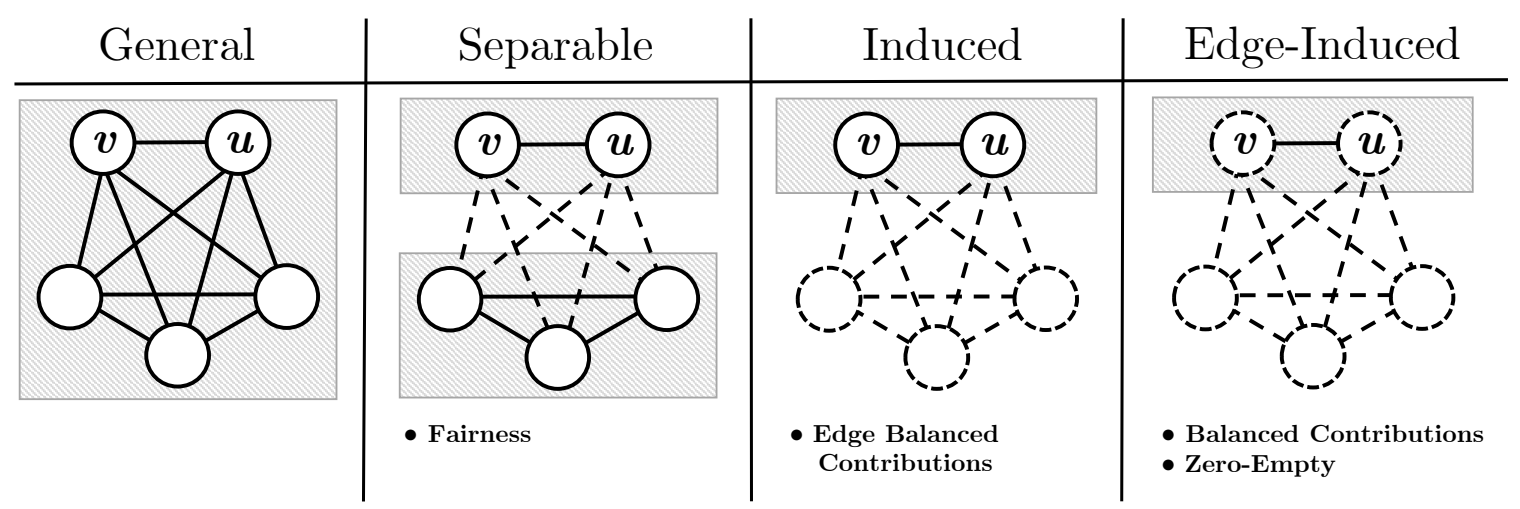

Figure 1: Given a complete graph of 5 nodes, the figure illustrates how the value of $\{v, u\}$ is computed under a general, a separable, an induced, and an edge-induced representation function. The gray boxes contain the edges and nodes that affect the value of $\{v, u\}$; all remaining edges and nodes (i.e., the dashed ones) do not. Since there are $2^{10}$ possible graphs consisting of 5 nodes each, $\{v, u\}$ may have at most $2^{10}$ distinct values under a general representation function, $r$. In contrast, if $r$ is separable, then $\{v, u\}$ may have at most $2^{4}$ distinct values, and if $r$ is induced or edge-induced, then $\{v, u\}$ may have at most $2^{1}$ distinct values. Below each graph, we list the axioms with which we characterise centralities in each class; see subsequent sections for more details.

- Separable representation functions (which produce separable game-theoretic centralities): in this class, the value of every subset of nodes depends solely on the subgraph induced by this subset and the subgraph induced by the remaining nodes in the graph.

- Induced representation functions (which produce induced game-theoretic centralities): in this class, the value of every subset of nodes depends solely on the subgraph induced by this subset. Hence, every induced game-theoretic centrality is also separable.

- Edge-induced representation functions (which produce edge-induced game-theoretic centralities): in this class, the value of every subset of nodes depends solely on the edges in the subgraph induced by this subset. ${ }^{2}$ Therefore, every edge-induced gametheoretic centrality is also induced.

Given a complete graph of 5 nodes, Figure 1 illustrates the edges that affect the value of a subset of nodes $(\{v, u\})$ under a general, a separable, an induced, and an edge-induced representation function.

Our analysis shows that each of the above classes of game-theoretic centralities captures a particular subset of all centralities. In particular, we first prove that the subset of all centralities that can be obtained using separable game-theoretic centralities is defined by Myerson's Fairness. In other words, every separable game-theoretic centrality satisfies Fairness, and every (non-game-theoretic) centrality that satisfies Fairness can be obtained with some separable game-theoretic centrality. Next, we extend Fairness to produce a new

2. Note that the difference between the induced and edge-induced representation functions is that the latter does not depend on isolated nodes, unlike the former one. 
axiom-Edge Balanced Contributions - which is a network counterpart of the aforementioned game-theoretic axiom of Balanced Contributions. Analogously, we prove that the subset of all centralities that can be obtained with induced game-theoretic centralities is defined by Edge Balanced Contributions. Finally, we prove that the subset of all centralities that can be obtained using edge-induced game-theoretic centralities is defined by two axioms: Myerson's Balanced Contributions axiom, and an additional axiom-Zero-Emptywhich simply requires that the centrality of any node in an empty graph (with no edges) is zero.

The remainder of the paper is structured as follows. Section 2 discusses the related works in the literature. Section 3 presents the necessary notation and background. Sections 4 to 7 analyse the general, separable, induced, and edge-induced game-theoretic centralities, respectively. Finally, Section 8 concludes the paper and presents some potential future directions. A summary of the main notation can be found in the appendix.

\section{Related Work}

In this section, we briefly review the literature on game-theoretic centralities, indicating which centralities could be classified as separable, induced, and edge-induced. We discuss, in particular, Attachment centrality - to date the only game-theoretic centrality the axiomatic foundations of which have been proposed. Finally, we comment on the characterisations of other, non-game theoretic centrality measures and other examples of applying coalitional games to networks.

\subsection{Game-Theoretic Network Centralities}

Game-theoretic centralities can be divided into two general types. In the first one, based on the seminal work by Myerson (1977), there is an explicit distinction between connected and disconnected coalitions. In the second type, there is no such distinction.

\subsubsection{Game-Theoretic Network Centralities Based on Connectivity}

For the first time coalitional games were considered on graphs in the seminal work by Myerson (1977). In particular, Myerson proposed a model of a coalitional game in which cooperation is restricted by a communication graph. The edges in this graph represent communication channels between players. The key idea behind Myerson's model is that only connected coalitions - i.e., coalitions in which each pair of nodes can communicate either directly (via an edge) or indirectly (via a path) - are able to coordinate their activities and generate an arbitrary payoff. As for any disconnected coalition, since the connected components therein cannot communicate with one another, the value of entire coalition is simply the sum of the values of the connected components therein.

The Shapley value of Myerson's graph-restricted game is called the Myerson value. The payoff assigned by the Myerson value to each player reflects the importance of this player in the graph-restricted game. Hence, it can be interpreted as a centrality index. Note that such centrality is determined not only by the topology of the graph but also by the definition of the coalitional game (i.e., the values of the different coalitions). For instance, Skibski, Michalak, Rahwan, and Wooldridge (2014) studied a family of game-theoretic centralities 
whereby the value of a coalition, $S$, in unweighted graphs simplifies to $|S|^{\alpha}|E(S)|^{\beta}$, where $\alpha$ and $\beta$ are constants, and $E(S)$ is the set of edges in the subgraph induced by $S$. Note that all centrality indices from this family belong to the class of induced game-theoretic centralities. Moreover, if $\alpha=0$, then all the resulting centrality indices belong to the class of edge-induced game-theoretic centralities.

Myerson (1977) proved that his value is characterised by two basic axioms: Fairness and Efficiency. As mentioned earlier in the introduction, Fairness requires that each edge in the graph equally affects the payoff of both adjacent players (see page 46 for a formal definition). On the other hand, Efficiency requires that the payoff of each connected component of a graph is entirely divided among the members of this component. While the former axiom can be easily translated to the context of network centrality, the latter cannot.

The first axiomatization of a game-theoretic centrality was proposed by Skibski et al. (2016). In their centrality, called Attachment centrality, the value of an arbitrary (not necessarily connected) coalition, $S$, is: $|S|-|K(G[S])|$, where $K(G[S])$ is the set of components of the subgraph induced by $S$. This implies that the value of a connected coalition, $S$, is: $|S|-1$. The authors proved that Attachment centrality is the only centrality satisfying the following four axioms: Locality, Normalization, Gain-loss, and Myerson's Fairness. In more detail, Locality concerns connected components of the graph; it requires that the centrality of a node depends solely on the connected component to which this node belongs. Normalization is inspired by the observation that most centrality measures (e.g., Degree, Closeness, and Betweenness) are minimized when the node is isolated (i.e., does not have any neighbours), and maximized when the node happens to be the center of a star. Hence, Normalization requires that a (normalized) centrality measure returns a minimum value of 0 , and a maximum value of $n-1$ at these extreme cases, respectively. Gain-Loss, just like Fairness, concerns the impact of adding an edge to the graph. However, while Fairness focuses on how this addition affects both ends of the edge, the other axiom focuses on how this addition affects every node other than the two ends of the edge. Specifically, according to Gain-Loss, if adding an edge in a connected graph makes some nodes' centrality greater, then it must also make some other nodes' centrality smaller, such that the sum of centralities remains unchanged. Arguably, Gain-loss makes sense from the connectivity point of view, since all nodes share the role of keeping the network connected. For example, consider a situation in which the removal of a node, $v$, breaks a connected graph, $G$, into two components, $G_{1}$ and $G_{2}$. Here, $v$ obviously plays an important role in terms of connectivity, since its presence is necessary to connect $G_{1}$ with $G_{2}$. Now suppose that the edge between nodes $u$ and $w$ was added to $G$, where $u$ belongs to $G_{1}$ and $w$ belongs to $G_{2}$. With this addition, it seems reasonable to claim that the role played by $u$ and $w$ grows more important, whereas the role played by $v$ diminishes, since its presence is no longer necessary to connect $G_{1}$ with $G_{2}$. Finally, note that Attachment centrality belongs to the class of edge-induced game-theoretic centralities.

Amer and Giménez (2004) proposed an alternative approach to the one proposed by Myerson. Specifically, the authors considered a connectivity game in which the value of each connected coalition is 1 , and the value of each disconnected coalition equals 0 . This definition was generalized by Lindelauf et al. (2013), who allowed the connected coalitions to have arbitrary values instead of just 1 . As an application, the authors considered the analysis of terrorist networks; here the value of every connected coalition was computed as 
the sum of the node weights. Finally, note that the centrality measure proposed by Amer and Giménez (2004), as well as all the centrality measures proposed by Lindelauf et al. (2013), all belong to the class of induced game-theoretic centralities.

\subsubsection{Game-Theoretic Network Centralities Not Requiring Connectivity}

In an attempt to identify the $k$ most influential nodes in the network, Narayanam and Narahari (2011) proposed a new centrality measure based on coalitional games. In this approach, the value of a coalition was given by the number of nodes in that coalition plus the number of nodes that are immediately reachable from that coalition, i.e., the neighbours. The centrality of each node was then computed as the Shapley value of that node in the coalitional game. Later on, Michalak et al. (2013) extended this work by considering five different functions for computing the value of each coalition; some of these functions deal with weighted graphs whereas the rest deal with unweighted graphs. All of these functions revolve around the idea that the value of a group is computed as the number of nodes in the group plus the number of nodes in a certain subset of nodes reachable from the group. Again, the centrality of each node was computed as the Shapley value of that node in the corresponding coalitional game. The centrality measures proposed by Michalak et al. (2013) and in the literature that followed (see www.game-theoretic-centrality.com) are not captured by any class proposed in this article - these measures do not belong even to our most general class, namely that of separable game-theoretic centralities.

Narayanam, Skibski, Lamba, and Michalak (2014) proposed two game-theoretic centrality measures in an attempt to determine the "gatekeepers" in a social network. In contrast to all other game-theoretic centrality measures in the literature, the authors considered two functions that compute the value of each coalition based on the network obtained by removing the coalition from the network. For instance, one such function computes the value of a coalition, $S$, as: $1 / \sum_{C \in K(G[V \backslash S])}|C|^{2}$, where $V$ is the set of nodes, and $K(G[V \backslash S])$ is the set of components of the subgraph induced by $V \backslash S$. With either function, the centrality of each node is computed as the Shapley value of that node in the corresponding coalitional game. The resulting centrality measures belong to the class of separable game-theoretic centralities, but do not belong to the class of induced game-theoretic centralities.

Moretti et al. (2010) considered a game-theoretic approach to measure the centrality of genes in networks, taking into consideration certain gene interactions. However, in their approach the value of a group of nodes depends not only on the graph itself, but also on the additional information - the connections to certain key genes.

Finally, a number of authors considered game-theoretic centralities for directed graphs (Gomez et al., 2003; del Pozo et al., 2011). However, no axiomatization has been proposed for any such centrality measure.

\subsection{Characterisations of Non Game-Theoretic Centralities}

There have been several attempts to provide an axiomatic characterization of the standard centrality measures. In this context, Sabidussi (1966) considered axiomatization of the class of all centrality measures. Nieminen (1974) proposed an axiomatization of Degree centrality. Boldi and Vigna (2014) derived an axiomatization of a certain version of Closeness centrality. 
More recently, Brandes et al. proposed an approach that can be positioned in-between the purely axiomatic and the purely conceptual ones (Brandes, 2014; Schoch \& Brandes, 2015).

There also exist some non-axiomatic characterizations of centrality measures. Two such characterizations are due to Borgatti (2005) and Borgatti and Everett (2006). In more detail, Borgatti (2005) focused on networks where something is flowing from node to node across the edges. In this context, he characterized the centrality measures by two factors:

- the type of the flow, which may be indivisible (e.g., like transporting a certain physical object), or simultaneous (e.g., like sending a message to all neighbours);

- the type of path, which may have repeating nodes, repeating edges, or no repetition.

Borgatti argued that all standard centrality measures can be classified according to these two factors. In contrast, Borgatti and Everett (2006) characterized the centrality measures in term of "cohesiveness". Specifically, they classify the centrality measures based on:

- the type of path that the node is involved in; such a path can either be radial (i.e., starts from the node), or medial (i.e., goes through the node);

- the way in which the centrality of a node is affected by the paths it is involved in; this can either be based on the number (or "volume") of those paths, or the length of those paths.

The above produces four classes of centrality measures. The authors show how many centrality indices can be classified into those four classes.

\subsection{Other Applications of Coalitional Games to Networks}

There is a vast body of literature in which cooperative game theory intersects with network theory which is not concerned with node centrality. For instance, Deng and Papadimitriou (1994) proposed an induced subgraph representation in which the value of a coalition is the sum of weights of edges between nodes from this coalition. Ieong and Shoham (2005) introduced marginal-contribution networks which can be understood as the generalization of induced subgraph representation to hypergraphs, i.e., graphs where edges can connect any number of nodes. Elkind and Wooldridge (2009) extended this representation to hedonic games. Brânzei and Larson (2009) proposed coalitional affinity games, where edges represent affinity relation and the value of a player in a coalition is the sum of weights of edges to other players from this coalition. Similarly, Brânzei and Larson (2011) considered social distance games in which the utility of a player in a coalition is the harmonic centrality (average inversed distance to others) in a graph induced by the coalition.

In all these concepts the value of a coalition depends solely on edges within the coalition, as in induced game-theoretic centralities that we propose in this paper.

\section{Preliminaries}

In this section, we provide the necessary background and notation from both graph theory and coalitional game theory. A summary of the main notation can be found in the appendix. 


\subsection{Graph Theory}

A graph (network) is a pair $G=(V, E)$, where $V$ is the set of nodes and $E$ is the set of edges. The edge between any two nodes, $v, u \in V$, will be denoted by $\{v, u\}$. We denote by $E_{v}$ the edges of node $v$ in $E$, i.e., $E_{v}=\{\{v, w\} \in E: w \in V\}$. Given a set of nodes, $V$, the set of all possible graphs will be denoted by $\mathcal{G}^{V}$. Furthermore, the set of all possible edges will be denoted by $\mathcal{E}^{V}$, i.e., $\mathcal{E}^{V}=\{S \subseteq V:|S|=2\}$. Note that we do not consider self-loops, i.e., edges of the form $(v, v): v \in V$.

For any subset of nodes, $S \subseteq V$, the subgraph of $G$ induced by $S$ is denoted by $G[S]$ and is defined as the graph whose set of nodes is $S$ and whose set of edges consists of every edge in $E$ of which both ends belong to $S$. Formally:

$$
G[S]=(S,\{\{v, u\} \in E: v, u \in S\}) .
$$

A subgraph is said to be connected if there exists a path between every pair of nodes in that subgraph. Furthermore, any such connected subgraph, $G[S]$, is said to be maximal if $G\left[S^{\prime}\right]$ is disconnected for all $S \subset S^{\prime}$. We will refer to each maximal connected subgraph as a component of $G$. Also, we will denote by $\mathcal{K}(G)$ the partition of $V$ in which every subset induces a component of $G$.

A centrality index is a function, $c: \mathcal{G}^{V} \rightarrow \mathbb{R}^{V}$, that assigns to every node $v \in V$ a real number reflecting the importance of $v$ in $G$; this number is called the centrality of $v$. Typically, the higher the centrality, the more important or central the node. Given a set of nodes, $V$, the set of all possible centrality indices is denoted by $\mathcal{C}^{V}$. For every $c \in \mathcal{C}^{V}$ and every $k \in \mathbb{R}$, we define the centrality index $(k \cdot c)$ as follows: $(k \cdot c)_{v}(G)=k \cdot c_{v}(G)$ for all $G \in \mathcal{G}^{V}$ and $v \in V$. Similarly, for every $c, c^{\prime} \in \mathcal{C}^{V}$, we define the centrality index $\left(c+c^{\prime}\right)$ as follows: $\left(c+c^{\prime}\right)_{v}(G)=c_{v}(G)+c_{v}^{\prime}(G)$ for all $G \in \mathcal{G}^{V}$ and $v \in V$.

\subsection{Coalitional Game Theory}

A game is a pair, $(N, f)$, where $N$ is the set of players and $f: 2^{N} \rightarrow \mathbb{R}$ is the characteristic function, which assigns to each subset of players a real number reflecting its importance. Any subset of players, $S \subseteq N$, is called a coalition, and $f(S)$ is called the value of coalition $S$. Typically, $f(\emptyset)=0$. Given a set of players, $N$, the set of all possible games is denoted by $\mathcal{F}^{N}$.

A solution concept, $\varphi: \mathcal{F}^{N} \rightarrow \mathbb{R}^{N}$, is a function that assigns a payoff to each player, $v$, in any given game $(N, f)$; this payoff is denoted by $\varphi_{v}(f)$. Given a set of players $N$, the set of all possible solution concepts is denoted by $\Phi^{N}$.

A fundamental class of solution concepts is Semivalues (Dubey, Neyman, \& Weber, 1981). Let $\beta:\{0, \ldots,|N|-1\} \rightarrow[0,1]$ be a function such that $\sum_{k=0}^{|N|-1} \beta(k)=1$. Every such $\beta$ defines a unique semivalue, $\varphi^{\beta}$, based on which the payoff of a player $v \in N$ is computed as follows:

$$
\varphi_{v}^{\beta}(f)=\sum_{S \subseteq N \backslash\{v\}} \frac{\beta(|S|)}{\left(\begin{array}{c}
|N|-1 \\
|S|
\end{array}\right)}(f(S \cup\{v\})-f(S)) .
$$

Here, the expression $f(S \cup\{v\})-f(S)$ is known as the marginal contribution of player $v$ to coalition $S$. We will write $\beta^{*}(k)$ as a shorthand notation for $\beta(k) /\left(\begin{array}{c}|N|-1 \\ k\end{array}\right)$. A semivalue 


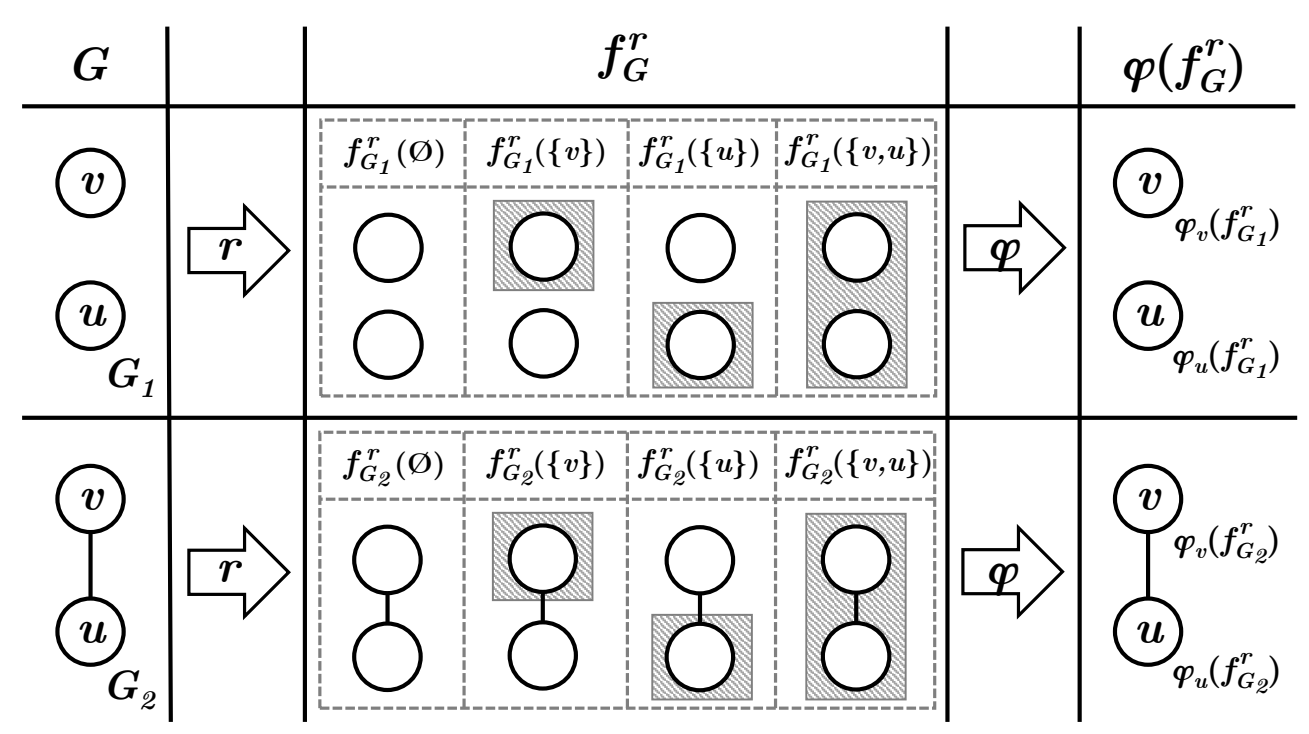

Figure 2: An illustration of a game-theoretic centrality, $(r, \varphi)$, on two sample networks, $G_{1}$ and $G_{2}$. For instance, given the graph $G_{1}=(V, E)$, the representation function, $r$, outputs a coalitional game, $\left(V, f_{G_{1}}^{r}\right)$. Then, the solution concept $\varphi$ assigns a payoff to every player in $V$; this payoff is interpreted as the centrality of the corresponding node.

is said to be positive if $\beta(k)>0$ for every $k \in\{0, \ldots,|N|-1\}$. Given $N$, the set of all semivalues is denoted by $\mathcal{S} \mathcal{V}^{N}$, and the set of all positive semivalues is denoted by $\mathcal{S} \mathcal{V}_{+}^{N}$. Thus, $\mathcal{S} \mathcal{V}_{+}^{N} \subseteq \mathcal{S} \mathcal{V}^{N} \subseteq \Phi^{N}$

Two well-known solution concepts, namely the Shapley value (Shapley, 1953) and the Banzhaf index (Banzhaf III, 1965), are in fact positive semivalues, with $\beta^{\text {Shapley }}(k)=1 /|N|$, and $\beta^{\text {Banzhaf }}(k)=\left(\begin{array}{c}|N|-1 \\ k\end{array}\right) / 2^{|N|-1}$.

\subsection{Game-Theoretic Centrality Indices}

We begin with the definition of a representation function, $r: \mathcal{G}^{V} \rightarrow \mathcal{F}^{V}$, which maps every graph, $G=(V, E)$, onto a cooperative game, $r(G)$, whose set of players is $V$, and whose characteristic function is denoted by $f_{G}^{r}$. That is to say, $r(G)=\left(V, f_{G}^{r}\right)$. Given a set of nodes $V$, the set of all possible representation functions will be denoted by $\mathcal{R}^{V}$.

A Game-Theoretic Centrality Index $(G T C)$ is a pair, $(r, \varphi)$, where $r$ is a representation function, and $\varphi$ is a solution concept. We say that a game-theoretic centrality index, $(r, \varphi)$, generates a centrality index, $[(r, \varphi)] \in \mathcal{C}^{V}$, computed for every $G \in \mathcal{G}^{V}$ and every $v \in V$ as follows:

$$
[(r, \varphi)]_{v}(G)=\varphi_{v}\left(f_{G}^{r}\right)
$$

In words, the centrality $[(r, \varphi)]$ of node $v$ in the graph $G$ equals the payoff of player $v$ in the game $r(G)$ according to the solution concept $\varphi$. Figure 2 illustrates the way this centrality is generated. This figure will be used in subsequent examples in the article. 
We say that $(r, \varphi)$ is based on $\varphi$. Given a set of nodes, $V$, the set of all game theoretic centrality indices will be denoted by $\mathcal{G} \mathcal{T} \mathcal{C}^{V}$. Formally:

$$
\mathcal{G} \mathcal{C}^{V}=\left\{(r, \varphi): r: \mathcal{G}^{V} \rightarrow \mathcal{F}^{V}, \varphi \in \Phi^{V}\right\}
$$

We will refer to $\mathcal{G} \mathcal{T} \mathcal{C}^{V}$ as the general class of game-theoretic centrality indices. For any given class, $\mathcal{I} \subseteq \mathcal{G} \mathcal{T} \mathcal{C}^{V}$, we will write $[\mathcal{I}]$ to denote the set of centrality indices generated by every $(r, \varphi) \in \mathcal{I}$. For instance, we have:

$$
\left[\mathcal{G} \mathcal{T C}{ }^{V}\right]=\left\{[(r, \varphi)]:(r, \varphi) \in \mathcal{G} \mathcal{T} \mathcal{C}^{V}\right\}
$$

Furthermore, for any given class, $\mathcal{I} \subseteq \mathcal{G} \mathcal{T} \mathcal{C}^{V}$, we will write $\mathcal{I}_{+}$to denote the subclass of $\mathcal{I}$ for which the solution concept happens to be a positive semivalue. Likewise, for any $\varphi \in \Phi^{V}$, we will write $\mathcal{I}_{\varphi}$ to denote the subclass of $\mathcal{I}$ for which the solution concept happens to be $\varphi$. For instance, we have:

$$
\begin{aligned}
& \mathcal{G T C}_{+}^{V}=\left\{(r, \varphi): r: \mathcal{G}^{V} \rightarrow \mathcal{F}^{V}, \varphi \in \mathcal{S V}_{+}^{V}\right\} \\
& \mathcal{G T C}_{\varphi}^{V}=\left\{(r, \varphi): r: \mathcal{G}^{V} \rightarrow \mathcal{F}^{V}\right\}
\end{aligned}
$$

In this article, we focus on $\mathcal{G} \mathcal{T} \mathcal{C}_{+}^{V}$. Furthermore, we restrict our attention to a fixed set of nodes, $V$, and so will often omit $V$ from notation such as $\mathcal{E}^{V}, \mathcal{C}^{V}, \Phi^{V}, \mathcal{S} \mathcal{V}^{V}$, and $\mathcal{G} \mathcal{C C}^{V}$. Finally, a summary of the main notation can be found in the appendix.

\section{General Class of Game-Theoretic Centralities}

At first glance, it may seem that $[\mathcal{G} \mathcal{T C}] \subsetneq \mathcal{C}$. However, as we will establish in Theorem 1 , for every centrality index, $c \in \mathcal{C}$, there exists a game-theoretic centrality index, $(r, \varphi)$, such that $[(r, \varphi)]=c$. The theorem builds upon a dummy game-a standard concept in cooperative game theory.

Theorem 1. For every positive semivalue $\varphi \in \mathcal{S} \mathcal{V}_{+}$,

$$
\left[\mathcal{G T C}_{\varphi}\right]=[\mathcal{G} \mathcal{T C}+]=[\mathcal{G T C}]=\mathcal{C}
$$

Proof. Since $\varphi \in \mathcal{S} \mathcal{V}_{+}$, then $\mathcal{G T C}_{\varphi} \subseteq \mathcal{G} \mathcal{T} \mathcal{C}_{+}$. Based on this, as well as the fact that $[\mathcal{G T C}] \subseteq \mathcal{C}$, we have:

$$
\left[\mathcal{G T C}_{\varphi}\right] \subseteq[\mathcal{G} \mathcal{T C}+] \subseteq[\mathcal{G T C}] \subseteq \mathcal{C}
$$

It remains to prove that $\mathcal{C} \subseteq\left[\mathcal{G T C}_{\varphi}\right]$. To put it differently, for every $c \in \mathcal{C}$, it remains to prove that there exists some $r \in \mathcal{R}$ such that $[(r, \varphi)]=c$. To this end, let $c \in \mathcal{C}$ be an arbitrary centrality index, and let us define a representation function $r(G)=\left(V, f_{G}^{r}\right)$ for every $G \in \mathcal{G}^{V}$ such that:

$$
\forall S \subseteq V, \quad f_{G}^{r}(S)=\sum_{v \in S} c_{v}(G)
$$

This is a dummy game - a game in which the value of every coalition is the sum of the values of its members. In our case, we set the value of every $v \in V$ to be equal to the centrality of $v$ in graph $G$ according to $c$. More precisely, we set $f_{G}^{r}(\{v\})=c_{v}(G)$. Now 
since the marginal contribution of $v$ to every coalition is equal to $c_{v}(G)$, then the definition of semivalues (Equation 1) implies that:

$$
\forall v \in V, \quad[(r, \varphi)]_{v}(G)=\varphi_{v}\left(f_{G}^{r}\right)=c_{v}(G)
$$

This concludes the proof of Theorem 1.

Let us illustrate the construction used in the proof of Theorem 1 using the networks from Figure 2 through the following example.

Example 1. Consider the degree centrality, $c^{D}$, defined as:

$$
c_{v}^{D}(G)=|\{\{v, u\} \in E: u \in V\}| .
$$

Now, given the two possible graphs in $\mathcal{G}^{\{v, u\}}$, i.e., given $G_{1}=(\{v, u\}, \emptyset)$ and $G_{2}=$ $(\{v, u\},\{\{v, u\}\})$, let us show how to generate $c^{D}$ using some GTC. First, let us deal with $G_{1}$. We need to specify $f_{G_{1}}^{r}$ such that $\varphi_{v}\left(f_{G_{1}}^{r}\right)=c_{v}^{D}\left(G_{1}\right)=0$, and $\varphi_{u}\left(f_{G_{1}}^{r}\right)=c_{u}^{D}\left(G_{1}\right)=0$. To this end, let us define a dummy game in which $f_{G_{1}}^{r}(\{v\})=c_{v}^{D}\left(G_{1}\right)=0$ and $f_{G_{1}}^{r}(\{u\})=$ $c_{u}^{D}\left(G_{1}\right)=0$. This implies that $f_{G_{1}}^{r}(\{v, u\})=f_{G_{1}}^{r}(\{v\})+f_{G_{1}}^{r}(\{u\})=0$. Since every marginal contribution of $v$ equals 0 (i.e., $f_{G_{1}}^{r}(\{v\})-f_{G_{1}}^{r}(\emptyset)=0$ and $\left.f_{G_{1}}^{r}(\{v, u\})-f_{G_{1}}^{r}(\{u\})=0\right)$, then from the definition of semivalues we get: $\varphi_{v}\left(f_{G_{1}}^{r}\right)=0$. Following the same reasoning, we get: $\varphi_{u}\left(f_{G_{1}}^{r}\right)=0$. Moving on to $G_{2}$, we define a dummy game in which $f_{G_{2}}^{r}(\{v\})=f_{G_{2}}^{r}(\{u\})=1$, which implies that $f_{G_{2}}^{r}(\{v, u\})=1+1=2$. Following the above reasoning, we get: $\varphi_{v}\left(f_{G_{2}}^{r}\right)=\varphi_{u}\left(f_{G_{2}}^{r}\right)=1$.

Next, we lay the theoretical foundation for the coming sections by showing that the totality of all centrality indices form a vector space. To this end, we will introduce the class of unanimity centrality indices.

Definition 1. (Unanimity Centrality Indices) Given a set of edges, $E^{\dagger} \subseteq \mathcal{E}^{V}$, and a set of nodes, $U \subseteq V, U \neq \emptyset$, the unanimity centrality index $c^{\left\langle U, E^{\dagger}\right\rangle}$ is defined for every $G=$ $(V, E) \in \mathcal{G}^{V}$ and every $v \in V$ as follows:

$$
c_{v}^{\left\langle U, E^{\dagger}\right\rangle}(G)= \begin{cases}1 & \text { if } v \in U \text { and } E^{\dagger} \subseteq E \\ 0 & \text { otherwise. }\end{cases}
$$

As such, $c^{\left\langle U, E^{\dagger}\right\rangle}$ assigns a value of 1 if and only if the node belongs to $U$ and the graph contains every edge from $E^{\dagger}$.

The set of all unanimity centrality indices will be denoted by $\mathcal{U}^{V}$, or simply $\mathcal{U}$ when there is no risk of confusion.

The next lemma provides a sufficient condition for the linear independence of the class of unanimity centrality indices.

Lemma 2. Let $\mathcal{U}^{*}$ be a set of unanimity centrality indices such that for every set of edges, $E^{\dagger} \subseteq \mathcal{E}^{V}$, and every pair, $c^{\left\langle U, E^{\dagger}\right\rangle}, c^{\left\langle U^{\prime}, E^{\dagger}\right\rangle} \in \mathcal{U}^{*}$, we have: $U=U^{\prime}$ or $U \cap U^{\prime}=\emptyset$. Then, $\mathcal{U}^{*}$ is linearly independent. 
Proof. If $\mathcal{U}^{*}=\emptyset$, then it is linearly independent. Now assume that $\mathcal{U}^{*} \neq \emptyset$ and that $\mathcal{U}^{*}$ is not linearly independent, i.e., there exists a collection of coefficients, $\alpha_{\left\langle U, E^{\dagger}\right\rangle}$ for every $c^{\left\langle U, E^{\dagger}\right\rangle} \in \mathcal{U}^{*}$, that are not all equal to zero, such that:

$$
\sum_{c^{\left\langle U, E^{\dagger}\right\rangle \in \mathcal{U}^{*}}}\left(\alpha_{\left\langle U, E^{\dagger}\right\rangle} \cdot c^{\left\langle U, E^{\dagger}\right\rangle}\right)_{v}(G)=0
$$

for every $v \in V$ and every $G \in \mathcal{G}^{V}$. This means that there exists a pair, $\left(U_{0}, E_{0}^{\dagger}\right)$, such that $\alpha_{\left\langle U_{0}, E_{0}^{\dagger}\right\rangle} \neq 0$ and $\alpha_{\left\langle U, E^{\dagger}\right\rangle}=0$ for every $E^{\dagger} \subsetneq E_{0}^{\dagger}$. Then, for $v \in U_{0}$, we have

$$
\begin{aligned}
\sum_{c^{\left\langle U, E^{\dagger}\right\rangle \in \mathcal{U}^{*}}}\left(\alpha_{\left\langle U, E^{\dagger}\right\rangle} \cdot c^{\left\langle U, E^{\dagger}\right\rangle}\right)_{v}\left(\left(V, E_{0}^{\dagger}\right)\right) & =\sum_{c^{\left\langle U, E^{\dagger}\right\rangle \in \mathcal{U}^{*}, E^{\dagger} \subseteq E_{0}^{\dagger}}}\left(\alpha_{\left\langle U, E^{\dagger}\right\rangle} \cdot c^{\left\langle U, E^{\dagger}\right\rangle}\right)_{v}\left(\left(V, E_{0}^{\dagger}\right)\right) \\
& =\sum_{c^{\left\langle U, E_{0}^{\dagger}\right\rangle} \in \mathcal{U}^{*}}\left(\alpha_{\left\langle U, E_{0}^{\dagger}\right\rangle} \cdot c^{\left\langle U, E_{0}^{\dagger}\right\rangle}\right)_{v}\left(\left(V, E_{0}^{\dagger}\right)\right) \\
& =\quad\left(\alpha_{\left\langle U_{0}, E_{0}^{\dagger}\right\rangle} \cdot c^{\left\langle U_{0}, E_{0}^{\dagger}\right\rangle}\right)_{v}\left(\left(V, E_{0}^{\dagger}\right)\right) \neq 0 .
\end{aligned}
$$

Here, we used the fact that $c_{v}^{\left\langle U, E^{\dagger}\right\rangle}((V, E))=0$ if $E^{\dagger} \nsubseteq E$ (Equation 3) and the fact that $c_{v}^{\left\langle U, E^{\dagger}\right\rangle}(G)=0$ if $v \notin U$ (Equations 4 and 5). This way, we obtain the desirable contradiction which concludes the proof of Lemma 2.

Next, we use Lemma 2 to characterize a basis of the class of all centrality indices, $\mathcal{C}$.

Theorem 3. The class $\mathcal{C}$ is a vector space with the basis:

$$
\mathcal{U}_{A l l}^{V}=\left\{c^{\left\langle\{v\}, E^{\dagger}\right\rangle}: v \in V, E^{\dagger} \subseteq \mathcal{E}^{V}\right\} .
$$

Proof. Since $\mathcal{C}$ is closed under addition (for every $c, c^{\prime} \in \mathcal{C}$ we have $c+c^{\prime} \in \mathcal{C}$ ) and closed under scalar multiplication (for every $c \in \mathcal{C}$ and a scalar $k \in \mathbb{R}$ we have $k \cdot c \in \mathcal{C}$ ), then $\mathcal{C}$ is a vector space. It remains to prove that $\mathcal{U}_{A l l}^{V}$ is a basis of $\mathcal{C}$. We know from Lemma 2 that $\mathcal{U}_{A l l}^{V}$ is linearly independent. Moreover, since $\left|\mathcal{U}_{A l l}^{V}\right|=|V \times \mathcal{E}|=|V \times \mathcal{G}|$, the size of $\mathcal{U}_{A l l}^{V}$ is the same as the dimension of $\mathcal{C}$. This concludes the proof.

The above result comes in handy when proving that all centrality indices from a given class can be generated with a subclass of GTCs. More in detail, the following lemma shows that if the basis of a class can be generated, then the whole class can also be generated.

Lemma 4. Let $\mathcal{C}^{*}$ be a class of centrality indices with a basis $\mathcal{U}^{*}$, and let $\mathcal{I} \subseteq \mathcal{G T C}_{\varphi}$ be a class of GTCs closed under addition and scalar multiplication. If $\mathcal{U}^{*} \subseteq[\mathcal{I}]$, then $\mathcal{C}^{*} \subseteq[\mathcal{I}]$.

Proof. Assume that $\mathcal{U}^{*}=\left\{c_{1}, c_{2}, \ldots, c_{m}\right\}$. Furthermore, for every $c_{i} \in \mathcal{U}^{*}$, let $\left(r_{i}, \varphi\right)$ denote a centrality from $\mathcal{I}$ such that $\left[\left(r_{i}, \varphi\right)\right]=c_{i}$. Since $\mathcal{U}^{*}$ is a basis of $\mathcal{C}^{*}$, every centrality $c \in \mathcal{C}^{*}$ is a linear combination of centralities from $\mathcal{U}^{*}$, i.e., there exists a collection of coefficients, $\alpha_{i}$ for every $c_{i}$, such that:

$$
c=\sum_{c_{i} \in \mathcal{U}^{*}} \alpha_{i} c_{i}
$$


From the additivity of semivalues, we have that $[(r, \varphi)]+\left[\left(r^{\prime}, \varphi\right)\right]=\left[\left(r+r^{\prime}, \varphi\right)\right]$ and $[(k$. $r, \varphi)]=k \cdot[(r, \varphi)]$ for arbitrary representation functions $r, r^{\prime}$, arbitrary scalar $k \in \mathbb{R}$, and arbitrary $\varphi \in \mathcal{S} \mathcal{V}_{+}$. Therefore, we have:

$$
\left[\left(\sum_{c_{i} \in \mathcal{U}^{*}} \alpha_{i} c_{i}, \varphi\right)\right]=c .
$$

Since we assumed that $\mathcal{I}$ is closed under addition and scalar multiplication, we know that this centrality belongs to $\mathcal{I}$. This concludes the proof of Lemma 4.

In the general class of game-theoretic centrality indices, for any two distinct graphs, $G, G^{\prime} \in \mathcal{G}$, a representation function $r$ may output two games, $\left(V, f_{G}^{r}\right),\left(V, f_{G^{\prime}}^{r}\right) \in \mathcal{F}$, that are completely independent from one another. In other words, the value of every subset of nodes $S \subseteq V$ under $f_{G}^{r}$ may be completely different than (or independent from) the value of the same subset under $f_{G^{\prime}}^{r}$. This implies that in order to define a game-theoretic centrality in the general form, one needs to specify all $2^{V}$ values of $f_{G}^{r}$ for every $G \in \mathcal{G}$. Such a centrality index would clearly be impractical. To overcome this limitation, every gametheoretic centrality index studied in the literature to date assumes some kind of dependency between $f_{G}^{r}$ and $f_{G^{\prime}}^{r}$ (see, e.g., Michalak et al., 2013). We follow this approach in the next sections, where we define three classes of game-theoretic centralities by imposing some natural requirements on the representation function.

\section{Separable Game-Theoretic Centralities}

The first subclass of $\mathcal{G} \mathcal{T C}$ that we consider is the class of separable game-theoretic centralities, or separable GTCs.

Definition 2. (Separable GTC) A representation function, $r$, is separable if for every coalition $S \subseteq V$ and every two graphs $G, G^{\prime} \in \mathcal{G}^{V}$ such that $G[S]=G^{\prime}[S]$ and $G[V \backslash S]=$ $G^{\prime}[V \backslash S]$ it holds that $f_{G}^{r}(S)=f_{G^{\prime}}^{r}(S)$. A GTC, $(r, \varphi)$, is separable if $r$ is separable. Given a set of nodes, $V$, the set of all separable GTCs is denoted by $\mathcal{S G T C}$.

In words, a game-theoretic centrality index is separable if the value of every coalition, $S \subseteq V$, under the representation function, $r$, depends solely on the subgraph induced by $S$ and the subgraph induced by $V \backslash S$ in $G$.

As we will show later on in this section, separable GTCs are related to the notion of Fairness, proposed by Myerson (1977).

Fairness: For every $G=(V, E)$ and every $v, u \in V$ such that $\{v, u\} \notin E$, adding the edge $\{v, u\}$ to the graph $G$ affects the centrality of $v$ and $u$ equally. Formally:

$$
c_{v}((V, E \cup\{\{v, u\}\}))-c_{v}((V, E))=c_{u}((V, E \cup\{\{v, u\}\}))-c_{u}((V, E)) .
$$

The class of all centralities satisfying Fairness will be denoted by $\mathcal{C}_{\text {Fair }}^{V}$, or simply $\mathcal{C}_{\text {Fair }}$ when there is no risk of confusion. 
For every node, $v \in V$, and edge, $e \in \mathcal{E}$, we will use the notation:

$$
\Delta_{v}^{c}(e, G)=c_{v}((V, E \cup\{e\}))-c_{v}((V, E)) .
$$

Note that, if $e \in E$, then $\Delta_{v}^{c}(e,(V, E))=0$. Using this notation, Fairness states that $\Delta_{v}^{c}(\{v, u\}, G)=\Delta_{u}^{c}(\{v, u\}, G)$ for every graph $G \in \mathcal{G}$ and $v, u \in V$.

The following lemma states that any centrality index in $\mathcal{C}_{\text {Fair }}$ can be uniquely characterized by only specifying the sum of node-centralities in every component of the graph (i.e., there is no need to specify the centrality of every node).

Lemma 5. For every function, $g: 2^{V} \times \mathcal{G}^{V} \rightarrow \mathbb{R}$, there exists at most ${ }^{3}$ one centrality index, $c \in \mathcal{C}_{\text {Fair }}^{V}$, that satisfies $\sum_{v \in S} c_{v}(G)=g(S, G)$ for every $G \in \mathcal{G}^{V}$ and $S \in \mathcal{K}(G)$.

Proof. Let $c, c^{\prime} \in \mathcal{C}^{V}$ be two centrality indices such that for every graph $G \in \mathcal{G}^{V}$ and every $S \in \mathcal{K}(G)$ we have:

$$
\sum_{v \in S} c_{v}(G)=g(S, G)=\sum_{v \in S} c_{v}^{\prime}(G)
$$

Then, it suffices to prove that $c_{v}(G)=c_{v}^{\prime}(G)$ for every graph $G \in \mathcal{G}^{V}$ and every $v \in V$. The proof will proceed by contradiction. Specifically, let $G=(V, E)$ be a graph with the minimum number of links such that $c_{v}(G) \neq c_{v}^{\prime}(G)$. Furthermore, let $e=\{v, u\} \in E$ be an arbitrary edge, and assume that $v, u$ belong to some $S \in \mathcal{K}(G)$. Based on Fairness, we have:

$$
\begin{aligned}
& c_{v}(G)-c_{u}(G)=c_{v}((V, E \backslash\{e\}))-c_{u}((V, E \backslash\{e\}))= \\
& c_{v}^{\prime}((V, E \backslash\{e\}))-c_{u}^{\prime}((V, E \backslash\{e\}))=c_{v}^{\prime}(G)-c_{u}^{\prime}(G) .
\end{aligned}
$$

Now, let $\delta_{v}=c_{v}(G)-c_{v}^{\prime}(G)$ for $v \in V$. We get:

$$
\delta_{v}=c_{v}(G)-c_{v}^{\prime}(G)=c_{u}(G)-c_{u}^{\prime}(G)=\delta_{u} .
$$

Consider a node $w \in S$. Since $v$ and $w$ are in the same component, then there exists a path $\left(v, u_{1}, u_{2}, \ldots, u_{k}, w\right)$ and we get:

$$
\delta_{v}=\delta_{u_{1}}=\ldots=\delta_{u_{k}}=\delta_{w} .
$$

Now, summing over all nodes from $S$ we get:

$$
|S| \cdot \delta_{v}=\sum_{w \in S} \delta_{w}=\sum_{w \in S}\left(c_{w}(G)-c_{w}^{\prime}(G)\right)=\sum_{w \in S} c_{w}(G)-\sum_{w \in S} c_{w}^{\prime}(G)=0 .
$$

Thus, $c_{v}(G)=c_{v}^{\prime}(G)$, which is a contradiction. This concludes the proof of Lemma 5 .

It should be noted that our proof of Lemma 5 follows the reasoning of Myerson (1977, Proof of Theorem, p.228). However, we could not use his result directly. In more details, Myerson proved that for every characteristic function $g: 2^{V} \rightarrow \mathbb{R}$ there exists a unique

3. While the lemma states that there exist at most one such centrality index, in the proof of Theorem 6 we prove that there exist exactly one. The same comment applies to Lemma 9 and Lemma 13 (in respect to Theorem 10 and 14). 
function, $c$ (in our work it is called "centrality", in Myerson's work it is called "allocation rule") that satisfies Fairness and satisfies: $\sum_{v \in S} c_{v}(G)=g(S)$ for every graph $G$ and every component $S \in \mathcal{K}(G)$. Our lemma shows that there is also at least one such function, if $g(S)$ depends on the graph.

Building upon Lemma 5 , the following theorem identifies a basis of the class $\mathcal{C}_{\text {Fair }}$.

Theorem 6. $\mathcal{C}_{\text {Fair }}$ is a vector space with the basis:

$$
\mathcal{U}_{\text {Fair }}^{V}=\left\{c^{\left\langle U, E^{\dagger}\right\rangle}: U \in \mathcal{K}\left(\left(V, E^{\dagger}\right)\right)\right\}
$$

Proof. We begin by showing that $\mathcal{C}_{\text {Fair }}$ is a vector space. To show that $\mathcal{C}_{\text {Fair }}$ is closed under addition, it suffices to observe that $\Delta_{v}^{c+c^{\prime}}(e, G)=\Delta_{v}^{c}(e, G)+\Delta_{v}^{c^{\prime}}(e, G)$ for every $v \in V, e \in \mathcal{E}$ and $c, c^{\prime} \in \mathcal{C}$. Therefore, if $c$ and $c^{\prime}$ satisfy Fairness, then $c+c^{\prime}$ also satisfies Fairness. Similarly, $\Delta_{v}^{k \cdot c}(e, G)=k \cdot \Delta_{v}^{c}(e, G)$ for every $v \in V, e \in \mathcal{E}, k \in \mathbb{R}$ and $c \in \mathcal{C}$. Thus, if $c$ satisfies Fairness, then $k \cdot c$ also satisfies Fairness. We proved that $\mathcal{C}_{F a i r}$ is closed under addition and scalar multiplication, therefore it is a vector space.

It remains to prove that $\mathcal{U}_{\text {Fair }}$ forms a basis of $\mathcal{C}_{\text {Fair }}$. To this end, we will show that $\mathcal{U}_{\text {Fair }} \subseteq \mathcal{C}_{\text {Fair }}$, that $\mathcal{U}_{\text {Fair }}$ is linearly independent, and that $\left|\mathcal{U}_{\text {Fair }}\right|$ is equal to the dimension of $\mathcal{C}_{\text {Fair. }}$.

First, let us show that $\mathcal{U}_{\text {Fair }} \subseteq \mathcal{C}_{\text {Fair }}$, i.e., the centralities from $\mathcal{U}_{\text {Fair }}$ satisfy Fairness. Let $c=c^{\left\langle U, E^{\dagger}\right\rangle} \in \mathcal{U}_{\text {Fair }}$ be an arbitrary centrality from $\mathcal{U}_{\text {Fair. }}$. Observe that $\Delta_{v}^{c}(\{v, u\}, G) \neq 0$ if and only if $v \in U$ and $E^{\dagger} \backslash E=\{\{v, u\}\}$ for $G=(V, E)$. In such a case, since $U \in \mathcal{K}\left(\left(V, E^{\dagger}\right)\right)$ induces a component of $G$ and $v \in U$, then $u \in U$, and $\Delta_{u}^{c}(\{v, u\}, G)=1=\Delta_{v}^{c}(\{v, u\}, G)$. Therefore, for every graph $G=(V, E) \in \mathcal{G}$ such that $\{v, u\} \notin E$, we have $\Delta_{v}^{c}(\{v, u\}, G)=$ $\Delta_{u}^{c}(\{v, u\}, G)$ and Fairness is satisfied.

Next, to show that $\mathcal{U}_{\text {Fair }}$ is linearly independent, we use Lemma 2: if $c^{\left\langle U, E^{\dagger}\right\rangle}, c^{\left\langle U^{\prime}, E^{\dagger}\right\rangle} \in$ $\mathcal{U}_{\text {Fair }}$, then $U, U^{\prime} \in \mathcal{K}\left(\left(V, E^{\dagger}\right)\right)$, therefore $U=U^{\prime}$ or $U \cap U^{\prime}=\emptyset$ and the condition sufficient for linear independence is met.

Finally, we show that $\left|\mathcal{U}_{\text {Fair }}\right|$ is equal to the dimension of $\mathcal{C}_{\text {Fair }}$. Let $\left|\mathcal{K}\left(\mathcal{G}^{V}\right)\right|$ denote the number of components in all graphs from $\mathcal{G}$, and let $\operatorname{dim}\left(\mathcal{C}_{\text {Fair }}\right)$ denote the dimension of $\mathcal{C}_{\text {Fair. }}$. From the definition, $\left|\mathcal{U}_{\text {Fair }}\right|=\left|\mathcal{K}\left(\mathcal{G}^{V}\right)\right|$. Since the centralities from $\mathcal{U}_{\text {Fair }}$ are linearly independent, we have: $\operatorname{dim}\left(\mathcal{C}_{\text {Fair }}\right) \geq\left|K\left(\mathcal{G}^{V}\right)\right|$. But from Lemma 5: $\operatorname{dim}\left(\mathcal{C}_{\text {Fair }}\right) \leq\left|\mathcal{K}\left(\mathcal{G}^{V}\right)\right|$. Thus, $\operatorname{dim}\left(\mathcal{C}_{\text {Fair }}\right)=\left|\mathcal{K}\left(\mathcal{G}^{V}\right)\right|=\left|\mathcal{U}_{\text {Fair }}\right|$. This concludes the proof of Theorem 6 .

Example 2. Consider the degree centrality $c^{D}$ from Example 1. Since adding an edge $\{v, u\}$ increases the centrality of both $v$ and $u$ by 1 , then $c^{D}$ satisfies Fairness. Consequently, we know from Theorem 6 that $c^{D}$ is a linear combination of unanimity centralities from $\mathcal{U}_{\text {Fair }}$. Let us generate $c^{D}$ using such a combination. To this end, consider $c^{\langle\{v, u\},\{\{v, u\}\}\rangle} \in \mathcal{U}_{\text {Fair }}$ for some arbitrary pair, $v, u \in V, v \neq u$. According to $c^{\langle\{v, u\},\{\{v, u\}\}\rangle}$, the centrality of $v$ and $u$ equals 1 if the edge $\{v, u\}$ belongs to the graph, otherwise the centrality of $v$ and $u$ equals 0. Summing over all such pairs, we get the degree centrality:

$$
c^{D}=\sum_{v, u \in V: v \neq u} c^{\langle\{v, u\},\{\{v, u\}\}\rangle} .
$$

Lemma 7. Every separable game-theoretic centrality from $\mathcal{G} \mathcal{T C}+$ satisfies Fairness. 
Proof. Let $(r, \varphi)$ be an arbitrary GTC such that $r$ is separable and $\varphi$ is a positive semivalue, based on function $\beta$. We will prove that $[(r, \varphi)]$ satisfies Fairness.

Let $G=(V, E)$ be an arbitrary graph and consider adding the edge $e=\{v, u\} \notin E$. Since $r$ is separable, $f_{G}^{r}(S)$ does not depend on edges between $S$ and $V \backslash S$. Thus, for a coalition $S \subseteq V$, we have

$$
f_{(V, E \cup\{e\})}^{r}(S)=f_{(V, E)}^{r}(S) \text { if }|S \cap\{v, u\}|=1 .
$$

Using Equation (1) we get:

$$
\begin{aligned}
& \quad[(r, \varphi)]_{v}((V, E \cup\{e\}))-[(r, \varphi)]_{v}((V, E))= \\
& \sum_{S \subseteq V, v, u \in S} \beta^{*}(|S|-1)\left(f_{(V, E \cup\{e\})}^{r}(S)-f_{(V, E)}^{r}(S)\right)-\sum_{S \subseteq V \backslash\{v, u\}} \beta^{*}(|S|)\left(f_{(V, E \cup\{e\})}^{r}(S)-f_{(V, E)}^{r}(S)\right) \\
& \quad=[(r, \varphi)]_{u}((V, E \cup\{e\}))-[(r, \varphi)]_{u}((V, E)) .
\end{aligned}
$$

This concludes the proof of Lemma 7.

We are ready to present our main result of this section. In the following theorem we state that the class of separable positive-semivalue based GTCs is characterized by Fairness.

Theorem 8. For every positive semivalue, $\varphi \in \mathcal{S V}_{+}$,

$$
\mathcal{C}_{\text {Fair }}=\left[\mathcal{S G T C}_{\varphi}\right]=\left[\mathcal{S G} \mathcal{T} \mathcal{C}_{+}\right]
$$

Proof. Since $\mathcal{S G T C}_{\varphi} \subseteq \mathcal{S G \mathcal { T C }}+$, Lemma 7 implies that:

$$
\left[\mathcal{S G}_{\mathcal{T}} \mathcal{C}_{\varphi}\right] \subseteq\left[\mathcal{S G} \mathcal{T} \mathcal{C}_{+}\right] \subseteq \mathcal{C}_{\text {Fair }}
$$

It remains to prove that for every $\varphi \in \mathcal{S} \mathcal{V}_{+}$, we have:

$$
\mathcal{C}_{\text {Fair }} \subseteq\left[\mathcal{S G T C}_{\varphi}\right]
$$

In words, we need to prove that every centrality $c$ that satisfies Fairness can be generated by $[(r, \varphi)]$ for some separable representation function, $r$. To this end, we first show that every centrality from $\mathcal{U}_{\text {Fair }}$ can be generated by $[(r, \varphi)]$ for some separable representation function $r$.

Let $c^{\left\langle U, E^{\dagger}\right\rangle} \in \mathcal{U}_{\text {Fair }}$ and $\varphi \in \mathcal{S} \mathcal{V}_{+}$be an arbitrary positive semivalue based on weights $\beta$. Consider a representation function, $r^{*}$, defined as follows:

$$
f_{(V, E)}^{r^{*}}(S)= \begin{cases}\frac{1}{\beta^{*}(|U|)+\beta^{*}(|U|-1)} & \text { if } S=U, E^{\dagger} \subseteq E \\ \frac{\beta^{*}(|U|)}{\left(\beta^{*}(|U|)+\beta^{*}(|U|-1)\right) \beta^{*}(|V|-1)} & \text { if } S=V, E^{\dagger} \subseteq E \\ 0 & \text { otherwise }\end{cases}
$$

First, we argue that $r^{*}$ is separable. To this end, let $G=(V, E)$ and $G^{\prime}=\left(V, E^{\prime}\right)$ be two graphs such that $G[S]=G^{\prime}[S]$ and $G[V \backslash S]=G^{\prime}[V \backslash S]$. We will prove that $f_{G}^{r^{*}}(S)=f_{G^{\prime}}^{r^{*}}(S)$ for every $S \subseteq V$. 
If $f_{G}^{r^{*}}(S) \neq 0$, then $E^{\dagger} \subseteq E$ and either $S=U$, or $S=V$. If $S=V$, then $G[S]=G^{\prime}[S]$ implies that $G=G^{\prime}$ and $f_{G}^{r^{*}}(S)=f_{G^{\prime}}^{r^{*}}(S)$. Now assume that $S=U$ and $E^{\dagger} \subseteq E$. We have that $G[U]=G^{\prime}[U]$ and $G[V \backslash U]=G^{\prime}[V \backslash U]$. Since $U$ is a component in the graph $\left(V, E^{\dagger}\right)$, edges from $E^{\dagger}$ are either in $G[U]$, or $G[V \backslash U]$. Thus, they are also in $G^{\prime}[U]$ and $G^{\prime}[V \backslash U]$. This implies that $E^{\dagger} \subseteq E^{\prime}$. Thus, we get $f_{G^{\prime}}^{r^{*}}(S)=1 /\left(\beta^{*}(|U|)+\beta^{*}(|U|-1)\right)=f_{G}^{r^{*}}(S)$.

We proved that if $f_{G}^{r^{*}}(S) \neq 0$, then $f_{G}^{r^{*}}(S)=f_{G^{\prime}}^{r^{*}}(S)$. Analogously, if $f_{G^{\prime}}^{r^{*}}(S) \neq 0$, then $f_{G_{*}}^{r^{*}}(S)=f_{G^{\prime}}^{r^{*}}(S)$. Therefore, $f_{G}^{r^{*}}(S)=0 \Leftrightarrow f_{G^{\prime}}^{r^{*}}(S)=0$, which concludes the proof that $f_{G}^{r^{*}}(S)=f_{G^{\prime}}^{r^{*}}(S)$ for every coalition $S$. Consequently, $r^{*}$ is separable.

Let us show that $\left[\left(r^{*}, \varphi\right)\right]=c^{\left\langle U, E^{\dagger}\right\rangle}$. Let $G=(V, E)$ be an arbitrary graph. If $E^{\dagger} \nsubseteq E$, then $f_{G}^{r^{*}}(S)=0$ for every $S \subseteq V$, and $\left[\left(r^{*}, \varphi\right)\right]_{v}(G)=0=c_{v}^{\left\langle U, E^{\dagger}\right\rangle}(G)$. Now assume that $E^{\dagger} \subseteq E$. Let us calculate the centrality $\left[\left(r^{*}, \varphi\right)\right]$ for node $v \in U$ :

$$
\begin{aligned}
{\left[\left(r^{*}, \varphi\right)\right]_{v}(G)=\sum_{S \subseteq V \backslash\{v\}} \beta^{*}(|S|)\left(f_{G}^{r^{*}}(S \cup\{v\})-f_{G}^{r^{*}}(S)\right) } & \\
& =\frac{\beta^{*}(|U|-1)+\frac{\beta^{*}(|V|-1) \beta(|U|)}{\beta^{*}(|V|-1)}}{\beta^{*}(|U|)+\beta^{*}(|U|-1)}=1=c_{v}^{\left\langle U, E^{\dagger}\right\rangle}(G) .
\end{aligned}
$$

Analogously, we calculate the centrality $\left[\left(r^{*}, \varphi\right)\right]$ for node $v \notin U$ :

$$
\begin{aligned}
{\left[\left(r^{*}, \varphi\right)\right]_{v}(G)=\sum_{S \subseteq V \backslash\{v\}} \beta^{*}(|S|)\left(f_{G}^{r^{*}}(S \cup\{v\})-f_{G}^{r^{*}}(S)\right) } & \\
& =\frac{-\beta^{*}(|U|)+\frac{\beta^{*}(|V|-1) \beta(|U|)}{\beta^{*}(|V|-1)}}{\beta^{*}(|U|)+\beta^{*}(|U|-1)}=0=c_{v}^{\left\langle U, E^{\dagger}\right\rangle}(G) .
\end{aligned}
$$

This concludes the proof that $\left[\left(r^{*}, \varphi\right)\right]=c^{\left\langle U, E^{\dagger}\right\rangle}$.

To conclude, we showed that for every $c^{\left\langle U, E^{\dagger}\right\rangle} \in \mathcal{U}_{\text {Fair }}$ there exists a separable representation function $r^{*}$ such that $\left[\left(r^{*}, \varphi\right)\right]=c^{\left\langle U, E^{\dagger}\right\rangle}$. The class of separable GTCs based on $\varphi$ is closed under addition and scalar multiplication, because if $r, r^{\prime}$ are separable, then $r+r^{\prime}$ and $k \cdot r$ for any $k \in \mathbb{R}$ are also separable. Thus, based on Lemma 4, every centrality from $\mathcal{C}_{\text {Fair }}$ can be generated by some centrality from $\mathcal{S} \mathcal{G} \mathcal{T C}_{\varphi}$. This concludes the proof of Theorem 8.

We end this section with an example showing how the degree centrality, $c^{D}$, can be generated from a separable GTC.

Example 3. The degree centrality satisfies Fairness, because $\Delta_{v}^{c^{D}}(\{v, u\},(V, E))=1$ for every $v \in V$ and every $\{v, u\} \notin E$. Therefore, we know from Theorem 8 that there exists a separable game-theoretic centrality index that generates $c^{D}$. Let us identify such a separable index. Note that the one used in Example 1 is not separable, because $f_{G_{1}}^{r}(\{v\})=0$ and $f_{G_{2}}^{r}(\{v\})=1$, while separability requires that $f_{G_{1}}^{r}(\{v\})=f_{G_{2}}^{r}(\{v\})$. Instead, consider the index $\left[\left(r^{D}, \varphi^{\text {Shapley }}\right)\right]$, where $\varphi^{\text {Shapley }}$ is the Shapley value, and $r^{D}$ is defined as: $f_{G}^{r^{D}}(S)=$ $2 \cdot|\{\{v, u\} \in E: v, u \in S\}|$. Given this $r^{D}$, we show that $\left[\left(r^{D}, \varphi^{\text {Shapley }}\right)\right]=c^{D}$.

To this end, we will use the four widely-known axioms that define the Shapley value, namely: Additivity, Null-player, Symmetry and Efficiency. ${ }^{4}$ First of all, observe that:

4. For more on the various axiomatizations of the Shapley value, see, e.g., the work by Maschler, Solan, and Zamir (2013). 
$f_{G}^{r^{D}}(S)=\sum_{e \in E} f_{(V,\{e\})}^{r^{D}}(S), \forall S \subseteq V$. Thus, based on the Additivity axiom, we have:

$$
\varphi_{v}^{\text {Shapley }}(G)=\sum_{e \in E} \varphi_{v}^{\text {Shapley }}((V,\{e\})) .
$$

This allows us to focus our analysis on a single-edge graph, $(V,\{e\})$. Let us focus on $G^{*}=\left(V,\left\{\left\{v_{1}, v_{2}\right\}\right\}\right)$. Here, it is clear from the definition of $f_{G^{*}}^{r^{D}}$ that the only two players with non-zero marginal contributions are $v_{1}$ and $v_{2}$. Thus, based on the Null-player axiom: $\varphi_{u}^{\text {Shapley }}\left(f_{G^{*}}^{r^{D}}\right)=0, \forall u \in V \backslash\left\{v_{1}, v_{2}\right\}$. As for $v_{1}$ and $v_{2}$, since they are symmetric, then based on the Symmetry axiom, we have: $\varphi_{v_{1}}^{\text {Shapley }}\left(f_{G^{*}}^{r^{D}}\right)=\varphi_{v_{2}}^{\text {Shapley }}\left(f_{G^{*}}^{r^{D}}\right)$. Finally, since $f_{G^{*}}^{r^{D}}(V)=2$, then based on the Efficiency axiom, we have: $\sum_{v \in V} \varphi_{v}^{\text {Shapley }}\left(f_{G^{*}}^{r^{D}}\right)=2$. We have shown that the payoffs of all nodes in $G^{*}$ add up to 2 , and that $v_{1}$ and $v_{2}$ have equal payoffs, whereas the remaining nodes have zero payoff each, implying that $\varphi_{v_{1}}^{\text {Shapley }}\left(f_{G^{*}}^{r^{D}}\right)=$ $\varphi_{v_{2}}^{\text {Shapley }}\left(f_{G^{*}}^{r^{D}}\right)=1$. This as well as Equation (7) imply that every edge in $G$ increases the payoff of each of its ends by 1 , which is precisely what degree centrality does. Thus, $\left[\left(r^{D}, \varphi^{\text {Shapley }}\right)\right]=c^{D}$.

Note that $r^{D}$ in Example 3 depends solely on $G[S]$. Such representation functions are the subject of the next section.

\section{Induced Game-Theoretic Centralities}

In this section, we define a subclass of separable GTCs which we call induced game-theoretic centralities, or induced GTCs.

Definition 3. (Induced GTC) A representation function, $r$, is induced if for every coalition $S \subseteq V$ and every two graphs $G, G^{\prime} \in \mathcal{G}^{V}$ such that $G[S]=G^{\prime}[S]$ it holds that $f_{G}^{r}(S)=$ $f_{G^{\prime}}^{r}(S)$. A GTC, $(r, \varphi)$, is induced if $r$ is induced. The set of all induced game-theoretic centralities is denoted by $\mathcal{I} \mathcal{G} \mathcal{T C}$.

In words, a GTC is induced if the value of a coalition $S$ in the representation function depends solely on the subgraph induced by $S$ in $G$. Thus, every induced GTC is separable.

Given eight different graphs, $G_{1}, \ldots, G_{8}$, consisting of three nodes each, Figure 3 illustrates the edges that affect the value of $\{v\}$ under a general, a separable, and an induced representation function.

To characterize the class of induced GTCs we introduce a new property that we call Edge Balanced Contributions.

Edge Balanced Contributions: For every $G=(V, E)$, and every $e=\{v, \widetilde{v}\}$, $e^{\prime}=\{u, \widetilde{u}\}, e, e^{\prime} \notin E$, adding $e^{\prime}$ affects the difference in centrality of $v$ caused by the addition of e in the same way that adding e affects the difference in centrality of $u$ caused by the addition of $e^{\prime}$. More formally:

$$
\Delta_{v}^{c}\left(e,\left(V, E \cup\left\{e^{\prime}\right\}\right)\right)-\Delta_{v}^{c}(e,(V, E))=\Delta_{u}^{c}\left(e^{\prime},(V, E \cup\{e\})\right)-\Delta_{u}^{c}\left(e^{\prime},(V, E)\right) .
$$

Consider Equation (8) in more detail. Fix an edge $e=\{v, \bar{v}\}$ and a centrality measure $c$. Let us associate with an edge the value $\Delta_{v}^{c}(e,(V, E))$, which is the effect of removing 


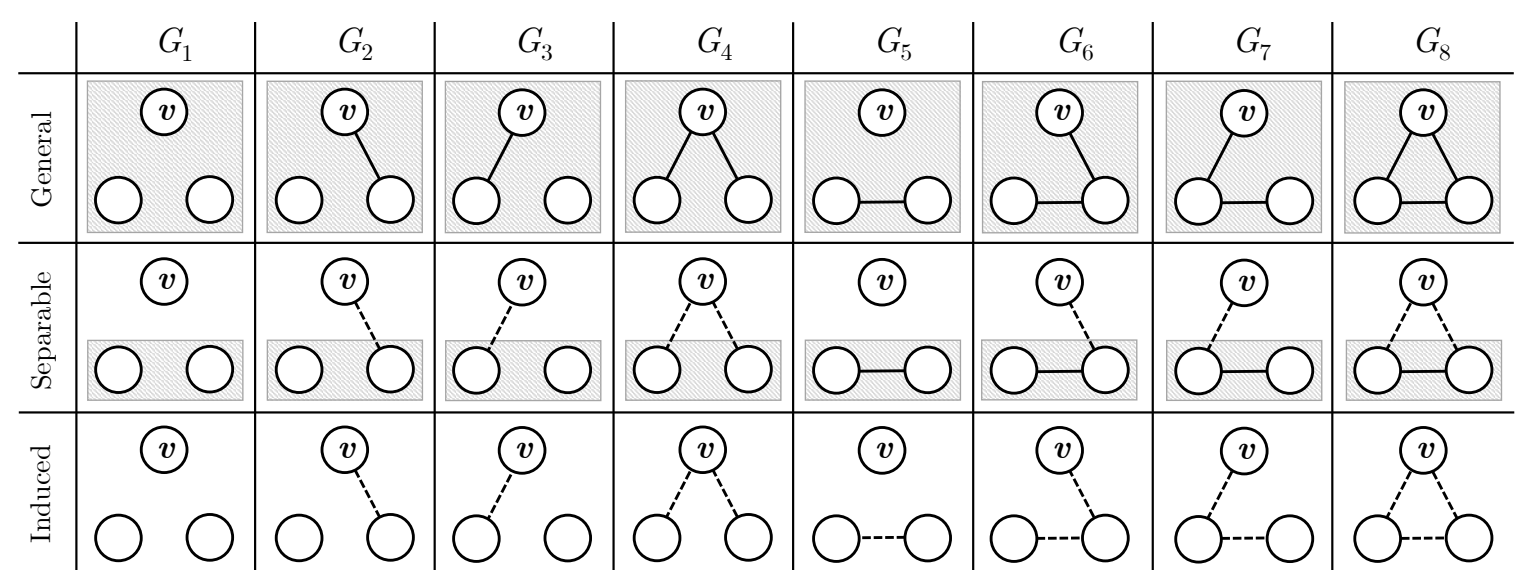

Figure 3: Given different graphs, $G_{1}, \ldots, G_{8}$, consisting of three nodes each, the figure illustrates how the value of $\{v\}$ is computed under a general, a separable, and an induced representation function. Specifically, the edges in the gray box affect the value of $\{v\}$, whereas the remaining (dashed) edges do not. Thus, under a general representation function, $r$, the coalition $\{v\}$ may have 8 distinct values in the graphs $G_{1}, \ldots, G_{8}$. In contrast, if $r$ is separable, then $\{v\}$ may have at most 2 distinct values: one in $G_{1}, \ldots, G_{4}$; the other in $G_{5}, \ldots, G_{8}$. Finally, if $r$ is induced, then $\{v\}$ can only have 1 value across all 8 graphs.

this edge from $(V, E)$ on the adjacent node. This value clearly depends on the graph; in particular, an existence of some other edge, $e^{\prime}=\{u, \bar{u}\}$, may have an impact on this value. This impact equals $\Delta_{v}^{c}\left(e,\left(V, E \cup\left\{e^{\prime}\right\}\right)\right)-\Delta_{v}^{c}(e,(V, E))$. Now, Edge Balanced Contribution states that the impact of edge $e^{\prime}$ on the value associated with edge $e$ is equal to the impact of edge $e$ on the value associated with edge $e^{\prime}$.

Given a set of nodes, $V$, the class of all centrality indices satisfying Edge Balanced Contributions will be denoted by $\mathcal{C}_{E B C}^{V}$, or just $\mathcal{C}_{E B C}$ when $V$ is clear from the context.

Recall from the introduction that this new property corresponds to the Balanced Contributions property, introduced by Myerson in the context of coalitional games (Myerson, 1980). Specifically, the Balanced Contributions property states that removing player $i$ from the game affects the payoff of player $j$ in the same way that removing player $j$ affects the payoff of player $i$. If we associate with removing of an edge the effect this removal has on both adjacent nodes, then Edge Balanced Contributions is an edge counterpart of Balanced Contributions.

Note that Edge Balanced Contributions implies Fairness. In particular, by setting $u=\widetilde{v}$ and $\widetilde{u}=v$, we have $e^{\prime}=e$, and we get $\Delta_{v}^{c}\left(e,\left(V, E \cup\left\{e^{\prime}\right\}\right)\right)=0$. Then, Equation (8) simplifies to: $-\Delta_{v}^{c}(\{v, u\},(V, E))=-\Delta_{u}^{c}(\{v, u\},(V, E))$ for every $\{v, u\} \notin E$, which is equivalent to Fairness.

Now, let $\mathcal{K}_{s}(G)$ be the set of isolated nodes, i.e., $\mathcal{K}_{s}(G)=\{v \in V:\{v\} \in \mathcal{K}(G)\}$, where the " $s$ " in $\mathcal{K}_{s}$ stands for "single-node". The following lemma states that any centrality index in $\mathcal{C}_{E B C}$ can be uniquely characterized by specifying (1) the centrality of every single-node component in $G$; and (2) the sum of node-centralities over all other components in $G$. 
Lemma 9. For every function, $g: 2^{V} \times \mathcal{G}^{V} \rightarrow \mathbb{R}$, there exists at most one centrality index, $c \in \mathcal{C}_{E B C}^{V}$ that satisfies $\sum_{v \in V \backslash \mathcal{K}_{s}(G)} c_{v}(G)=g\left(V \backslash \mathcal{K}_{s}(G), G\right)$ and $c_{v}(G)=g(\{v\}, G)$ for every $G \in \mathcal{G}^{V}$ and every $v \in \mathcal{K}_{s}(G)$.

Proof. Let $c, c^{\prime} \in \mathcal{C}^{V}$ be two centrality indices such that for every graph $G \in \mathcal{G}^{V}$ :

$$
\begin{aligned}
& c_{v}(G)=g(\{v\}, G)=c_{v}^{\prime}(G) \text { for every } v \in \mathcal{K}_{s}(G) \text {, and } \\
& \qquad \sum_{v \in V \backslash \mathcal{K}_{s}(G)} c_{v}(G)=g\left(V \backslash \mathcal{K}_{s}(G), G\right)=\sum_{v \in V \backslash \mathcal{K}_{s}(G)} c_{v}^{\prime}(G) .
\end{aligned}
$$

Then, it suffices to prove that $c_{v}(G)=c_{v}^{\prime}(G)$ for every graph $G \in \mathcal{G}^{V}, v \in V$.

Let $G=(V, E)$ be a graph with the minimum number of links such that $c(G) \neq c^{\prime}(G)$. If $E=\emptyset$ we get a contradiction, since $\mathcal{K}_{s}(G)=V$ and from the assumption $c_{v}(G)=c_{v}^{\prime}(G)$ for every $v \in \mathcal{K}_{s}(G)$. Assume $E \neq \emptyset$. Let $e=\{v, \widetilde{v}\}, e^{\prime}=\{u, \widetilde{u}\} \in E$ be two arbitrary edges (possible equal). Based on the Edge Balanced Contributions property of $c$, we have:

$\Delta_{v}^{c}(e,(V, E \backslash\{e\}))-\Delta_{u}^{c}\left(e^{\prime},\left(V, E \backslash\left\{e^{\prime}\right\}\right)\right)=\Delta_{v}^{c}\left(e,\left(V, E \backslash\left\{e, e^{\prime}\right\}\right)\right)-\Delta_{u}^{c}\left(e^{\prime},\left(V, E \backslash\left\{e, e^{\prime}\right\}\right)\right)$,

and further

$$
\begin{aligned}
c_{v}(G)-c_{u}(G)=c_{v}((V, E \backslash\{e\}))-c_{u}( & \left.\left(V, E \backslash\left\{e^{\prime}\right\}\right)\right) \\
& +\Delta_{v}^{c}\left(e,\left(V, E \backslash\left\{e, e^{\prime}\right\}\right)\right)-\Delta_{u}^{c}\left(e^{\prime},\left(V, E \backslash\left\{e, e^{\prime}\right\}\right)\right) .
\end{aligned}
$$

Performing the same calculations for $c^{\prime}$, and using the assumption that $G$ has a minimal number of links such that $c(G) \neq c^{\prime}(G)$ we have that:

$$
c_{v}(G)-c_{v}^{\prime}(G)=c_{u}(G)-c_{u}^{\prime}(G)
$$

Now, let $\delta_{v}=c_{v}(G)-c_{v}^{\prime}(G)$ for $v \in V$. Hence, we get $\delta_{v}=\delta_{u}$ for arbitrary two nodes $v, u$ with an edge. Summing over all such nodes, i.e., nodes from $V \backslash \mathcal{K}_{s}(G)$, we get:

$$
\left(|V| \backslash\left|\mathcal{K}_{s}(G)\right|\right) \cdot \delta_{v}=\sum_{w \in V \backslash \mathcal{K}_{s}(G)} \delta_{w}=\sum_{w \in V \backslash \mathcal{K}_{s}(G)} c_{w}(G)-\sum_{w \in V \backslash \mathcal{K}_{s}(G)} c_{w}^{\prime}(G)=0 .
$$

Thus, $\delta_{v}=0$ and $c_{v}(G)=c_{v}^{\prime}(G)$ for every $v \in V \backslash \mathcal{K}_{s}(G)$. From the assumption that $c_{v}(G)=c_{v}^{\prime}(G)$ for every $v \in \mathcal{K}_{s}(G)$ we get $c(G)=c^{\prime}(G)$, which is a contradiction. This concludes the proof of Lemma 9 .

Building upon the above lemma, the following theorem identifies a basis of the class $\mathcal{C}_{E B C}$, i.e., the class of all centrality indices that satisfy Edge Balanced Contributions.

Theorem 10. $\mathcal{C}_{E B C}$ is a vector space with the basis:

$$
\mathcal{U}^{E B C}=\left\{c^{\left\langle\{v\}, E^{\dagger}\right\rangle}: v \in \mathcal{K}_{s}\left(\left(V, E^{\dagger}\right)\right)\right\} \cup\left\{c^{\left\langle U, E^{\dagger}\right\rangle}: U=V \backslash K_{s}\left(\left(V, E^{\dagger}\right)\right)\right\} .
$$


Proof. Since $\Delta_{v}^{c}(e, G)$ is a linear function, then if $c$ and $c^{\prime}$ satisfy Edge Balanced Contributions, then $c+c^{\prime}$ and $k \cdot c$ satisfies Edge Balanced Contributions, too, for every $k \in \mathbb{R}$. Thus, $\mathcal{C}_{E B C}$ is a vector space.

To prove that $\mathcal{U}_{E B C}$ forms a basis of $\mathcal{C}_{E B C}$ we follow a reasoning similar to the one in the proof of Theorem 6: we show that $\mathcal{U}_{E B C} \subseteq \mathcal{C}_{E B C}$, that $\mathcal{U}_{E B C}$ is linearly independent, and that $\left|\mathcal{U}_{E B C}\right|$ is equal to the dimension of $\mathcal{C}_{E B C}$.

First, let us show that $\mathcal{U}_{E B C} \subseteq \mathcal{C}_{E B C}$, i.e., centralities from $\mathcal{U}_{E B C}$ satisfy Edge Balanced Contributions. To this end, let $c=c^{\left\langle U, E^{\dagger}\right\rangle} \in \mathcal{U}_{E B C}$ be an arbitrary centrality from $\mathcal{U}_{E B C}$. Consider the condition when:

$$
\Delta_{v}^{c}\left(e,\left(V, E \cup\left\{e^{\prime}\right\}\right)\right)-\Delta_{v}^{c}(e,(V, E)) \neq 0 .
$$

The value $\Delta_{v}^{c}(e,(V, E)) \neq 0$ if and only if $v \in U$ and $e \in E^{\dagger}$ and $(V, E)$ contains all edges from $E^{\dagger}$ apart of $e$, i.e., $E^{\dagger} \backslash E=\{e\}$. Therefore, Equation (9) holds only if $v \in U$, $E^{\dagger} \backslash\left(E \cup\left\{e^{\prime}\right\}\right)=\{e\}$, but $E^{\dagger} \backslash E \neq\{e\}$. It means that $e^{\prime} \in E^{\dagger}$. Summing up, Equation (9) is satisfied if $E^{\dagger} \backslash E=\left\{e, e^{\prime}\right\}$ and $v \in U$. In such a case, $\Delta_{v}^{c}\left(e,\left(V, E \cup\left\{e^{\prime}\right\}\right)\right)-\Delta_{v}^{c}(e,(V, E))=1$. But from the definition of $\mathcal{U}_{E B C}$ if $v \in U$ and $v$ has an edge in $E^{\dagger}$, then $U=V \backslash \mathcal{K}_{s}(G)$, i.e., contains all nodes with edges. Thus, nodes from $e^{\prime}$ also belong to $U$, and $\Delta_{u}^{c}\left(e^{\prime},(V, E \cup\right.$ $\{e\}))-\Delta_{v}^{c}\left(e^{\prime},(V, E)\right)=1$ for $u \in e^{\prime}$. Conversely, if Equation (9) does not hold, then condition from Equation (8) is also satisfied. This concludes the proof that all centralities from $\mathcal{U}_{E B C}$ satisfy Edge Balanced Contributions.

To see that $\mathcal{U}_{E B C}$ is linearly independent observe that if $c^{\left\langle U, E^{\dagger}\right\rangle}, c^{\left\langle U^{\prime}, E^{\dagger}\right\rangle} \in \mathcal{U}_{E B C}(U \neq$ $U^{\prime}$ ), then $U \cap U^{\prime}=\emptyset$. Therefore, Lemma 2 implies linear independence.

Finally, we show that $\left|\mathcal{U}_{E B C}\right|$ is equal to the dimension of $\mathcal{C}_{E B C}$. Note that $\left|\mathcal{U}_{E B C}\right|$ equals the number of one-node components in all graphs from $\mathcal{G}$ plus the number of graphs from $\mathcal{G}$ with at least one edge. Since the centralities from $\mathcal{U}_{E B C}$ are linearly independent, the dimension of the class $\mathcal{C}_{E B C}$ is at least equal $\left|\mathcal{U}_{E B C}\right|$. But from Lemma 9 we know that it is equal at most $\left|\mathcal{U}_{E B C}\right|$. Thus, the dimension of $\mathcal{C}_{E B C}$ equals $\left|\mathcal{U}_{E B C}\right|$. This concludes the proof of Theorem 10.

Lemma 11. Every induced game-theoretic centrality index in $\mathcal{G} \mathcal{T C}+$ satisfies Edge Balanced Contributions.

Proof. Let $(r, \varphi)$ be an arbitrary GTC such that $r$ is induced and $\varphi$ is a positive semivalue based on some $\beta$. We will prove that $[(r, \varphi)]$ satisfies Edge Balanced Contributions.

Let $G=(V, E)$ be an arbitrary graph and consider adding the edge $e=\{v, \widetilde{v}\} \notin E$. Since $r$ is induced, $f_{G}^{r}(S)$ does not depend on edges outside of $G[S]$. Thus, for a coalition $S \subseteq V$, we have:

$$
f_{(V, E \cup\{e\})}^{r}(S)=f_{(V, E)}^{r}(S) \text { if }\{v, \widetilde{v}\} \nsubseteq S .
$$

Using Equation (1) we get:

$$
\begin{aligned}
\Delta_{v}^{[(r, \varphi)]}(e,(V, E))=[(r, \varphi)]_{v}(V, E \cup\{e\})-[(r, \varphi)]_{v}(V, E) \\
\quad=\sum_{S \subseteq V: v, \widetilde{v} \in S} \beta^{*}(|S|-1)\left(f_{(V, E \cup\{e\})}^{r}(S)-f_{(V, E)}^{r}(S)\right) .
\end{aligned}
$$


Consider the left-hand side of the condition of Edge Balanced Contributions (Equation 8):

$$
\begin{aligned}
& \Delta_{v}^{[(r, \varphi)]}\left(e,\left(V, E \cup\left\{e^{\prime}\right\}\right)\right)-\Delta_{v}^{[(r, \varphi)]}(e,(V, E)) \\
& \quad=\sum_{S \subseteq V: v, \widetilde{v} \in S} \beta^{*}(|S|-1)\left(f_{\left(V, E \cup\left\{e, e^{\prime}\right\}\right)}^{r}(S)-f_{\left(V, E \cup\left\{e^{\prime}\right\}\right)}^{r}(S)-f_{(V, E \cup\{e\})}^{r}(S)+f_{(V, E)}^{r}(S)\right) .
\end{aligned}
$$

Now, let $e^{\prime}=\{u, \widetilde{u}\}$. Note that if $u, \widetilde{u} \notin S$, then the following holds: $f_{\left(V, E \cup\left\{e, e^{\prime}\right\}\right)}^{r}(S)=$ $f_{(V, E \cup\{e\})}^{r}(S)$ and $f_{\left(V, E \cup\left\{e^{\prime}\right\}\right)}^{r}(S)=f_{(V, E)}^{r}(S)$. Therefore:

$$
\begin{aligned}
& \Delta_{v}^{[(r, \varphi)]}\left(e,\left(V, E \cup\left\{e^{\prime}\right\}\right)\right)-\Delta_{v}^{[(r, \varphi)]}(e,(V, E)) \\
& =\sum_{S \subseteq V: v, \widetilde{v}, u, \widetilde{u} \in S} \beta^{*}(|S|-1)\left(f_{\left(V, E \cup\left\{e, e^{\prime}\right\}\right)}^{r}(S)-f_{\left(V, E \cup\left\{e^{\prime}\right\}\right)}^{r}(S)-f_{(V, E \cup\{e\})}^{r}(S)+f_{(V, E)}^{r}(S)\right) \\
& \quad=\Delta_{u}^{[(r, \varphi)]}\left(e^{\prime},(V, E \cup\{e\})\right)-\Delta_{u}^{[(r, \varphi)]}\left(e^{\prime},(V, E)\right) .
\end{aligned}
$$

This concludes the proof of Lemma 11.

Finally, as the main result of this section, we prove that the class of induced positive semivalue-based GTCs is characterized by the property of Edge Balanced Contributions.

Theorem 12. For every positive semivalue, $\varphi \in \mathcal{S} \mathcal{V}_{+}$,

$$
\mathcal{C}_{E B C}=\left[\mathcal{I} \mathcal{G} \mathcal{T} \mathcal{C}_{\varphi}\right]=[\mathcal{I} \mathcal{G} \mathcal{T C}+]
$$

Proof. Since $\mathcal{I G T C}_{\varphi} \subseteq \mathcal{I} \mathcal{G} \mathcal{T} \mathcal{C}_{+}$, Lemma 11 implies that:

$$
\left[\mathcal{I G T C}_{\varphi}\right] \subseteq[\mathcal{I} \mathcal{G} \mathcal{T C}+] \subseteq \mathcal{C}_{E B C}
$$

It remains to prove that for every $\varphi \in \mathcal{S} \mathcal{V}_{+}$, we have:

$$
\mathcal{C}_{E B C} \subseteq\left[\mathcal{I} \mathcal{G} \mathcal{T} \mathcal{C}_{\varphi}\right]
$$

i.e., that every centrality, $c$, satisfying Edge Balanced Contributions can be generated by $[(r, \varphi)]$ for some induced representation function, $r$.

We begin by showing that every unanimity centrality $c^{\left\langle U, E^{\dagger}\right\rangle}$ such that every edge from $E^{\dagger}$ is between nodes from $U$ (i.e., $\{v, u\} \in E^{\dagger}$ implies $v, u \in U$ ) can be generated by an induced game-theoretic centrality from $\mathcal{G} \mathcal{T} \mathcal{C}_{+}$.

Let $\varphi \in \mathcal{S} \mathcal{V}_{+}$be an arbitrary positive semivalue based on weights $\beta$. Consider a representation function, $r^{\left\langle U, E^{\dagger}\right\rangle}$, defined as follows:

$$
f_{(V, E)}^{r\left\langle U, E^{\dagger}\right\rangle}(S)= \begin{cases}\left(\sum_{k=|U|}^{|V|}\left(\begin{array}{c}
|V|-|U| \\
k-|U|
\end{array}\right) \beta^{*}(k-1)\right)^{-1} & \text { if } U \subseteq S, E^{\dagger} \subseteq E, \\
0 & \text { otherwise. }\end{cases}
$$

Since all edges from $E^{\dagger}$ are between nodes from $U$, and $U \subseteq S$, then $f_{G}^{\gamma^{\left\langle U, E^{\dagger}\right\rangle}}(S)$ depends solely on $G[S]$, which means that $r^{\left\langle U, E^{\dagger}\right\rangle}$ is induced.

Now, let us show that $\left[\left(r^{\left\langle U, E^{\dagger}\right\rangle}, \varphi\right)\right]=c^{\left\langle U, E^{\dagger}\right\rangle}$. To this end, let $G=(V, E) \in \mathcal{G}^{V}$ be an arbitrary graph. If $E^{\dagger} \nsubseteq E$, then $r_{G}^{\left\langle U, E^{\dagger}\right\rangle}(S)=0$ for every $S \subseteq V$, and $\left[\left(r^{\left\langle U, E^{\dagger}\right\rangle}, \varphi\right)\right]_{v}(G)=$ 
$0=c_{v}^{\left\langle U, E^{\dagger}\right\rangle}(G)$ for every $v \in V$. Now assume that $E^{\dagger} \subseteq E$. Let us calculate the centrality $\left[\left(r^{\left(\left\langle U, E^{\dagger}\right\rangle\right.}, \varphi\right)\right]$ for node $v \in U$ :

$$
\begin{aligned}
{\left[\left(r^{\left\langle U, E^{\dagger}\right\rangle}, \varphi\right)\right]_{v}(G)=} & \sum_{S \subseteq V \backslash\{v\}} \beta^{*}(|S|)\left(f_{G}^{r^{\left\langle U, E^{\dagger}\right\rangle}}(S \cup\{v\})-f_{G}^{r^{\left\langle U, E^{\dagger}\right\rangle}}(S)\right) \\
= & \sum_{S \subseteq V \backslash\{v\}: U \backslash\{v\} \subseteq S} \beta^{*}(|S|)\left(\sum_{k=|U|}^{|V|}\left(\begin{array}{c}
|V|-|U| \\
k-|U|
\end{array}\right) \beta^{*}(k-1)\right)^{-1} \\
& =\frac{\sum_{k=|U|}^{|V|}\left(\begin{array}{c}
|V|-|U| \\
k-|U|
\end{array}\right) \beta^{*}(k-1)}{\sum_{k=|U|}^{|V|}\left(\begin{array}{c}
|V|-|U| \\
k-|U|
\end{array}\right) \beta^{*}(k-1)}=1=c_{v}^{\left\langle U, E^{\dagger}\right\rangle}(G) .
\end{aligned}
$$

If $v \notin U$, then $v$ has zero marginal contribution to every coalition and $\left[\left(r^{\left\langle U, E^{\dagger}\right\rangle}, \varphi\right)\right]_{v}(G)=$ $0=c_{v}^{\left\langle U, E^{\dagger}\right\rangle}(G)$. This concludes the proof that $\left[\left(r^{\left\langle U, E^{\dagger}\right\rangle}, \varphi\right)\right]=c^{\left\langle U, E^{\dagger}\right\rangle}$.

Let us go back to showing that every unanimity centrality from $\mathcal{U}_{E B C}$ can be generated by $[(r, \varphi)]$ for some induced representation function, $r$. Let $\left(V, E^{\dagger}\right) \in \mathcal{G}^{V}$ be an arbitrary graph. Recall that if $c^{\left\langle U, E^{\dagger}\right\rangle} \in \mathcal{U}_{E B C}$ then either $U=V \backslash \mathcal{K}_{s}(G)$, or $U=\{v\}$ and $v \in \mathcal{K}(G)$. In the first case, we already proved that $\left[\left(r^{\left\langle U, E^{\dagger}\right\rangle}, \varphi\right)\right]=c^{\left\langle U, E^{\dagger}\right\rangle}$. To generate $c^{\left\langle\{v\}, E^{\dagger}\right\rangle}$ we use the following centrality:

$$
\begin{aligned}
{\left[\left(r^{\left\langle\left(V \backslash \mathcal{K}_{s}(G)\right) \cup\{v\}, E^{\dagger}\right\rangle}-r^{\left\langle\left(V \backslash \mathcal{K}_{s}(G)\right), E^{\dagger}\right\rangle}, \varphi\right)\right] } & =\left[\left(r^{\left\langle\left(V \backslash \mathcal{K}_{s}(G)\right) \cup\{v\}, E^{\dagger}\right\rangle}, \varphi\right)\right]-\left[\left(r^{\left\langle V \backslash \mathcal{K}_{s}(G), E^{\dagger}\right\rangle}, \varphi\right)\right] \\
& =c^{\left.\left\langle V \backslash \mathcal{K}_{s}(G)\right) \cup\{v\}, E^{\dagger}\right\rangle}-c^{\left\langle V \backslash \mathcal{K}_{s}(G), E^{\dagger}\right\rangle}=c^{\left\langle\{v\}, E^{\dagger}\right\rangle} .
\end{aligned}
$$

The class of induced GTCs based on $\varphi$ is closed under addition and scalar multiplication, because if $r, r^{\prime}$ are induced, then $r+r^{\prime}$ and $k \cdot r$ for every $k \in \mathbb{R}$ are also induced. This concludes the proof that for every $c^{\left\langle U, E^{\dagger}\right\rangle} \in \mathcal{U}_{E B C}$ there exists an induced GTCs that generates it. This fact, combined with Lemma 4 imply that every centrality from $\mathcal{C}_{E B C}$ can be generated by some centrality from $\mathcal{I G T C}_{\varphi}$. This concludes the proof of Theorem 12.

We end this section with an example showing that the flow betweenness centrality can be generated from a separable GTC.

Example 4. Consider the flow betwenness centrality (Freeman, Borgatti, \& White, 1991) defined as follows:

$$
c_{v}^{F}(G)=\sum_{s, t \in V}\left(\text { flow }_{s, t}(G)-\text { flow }_{s, t}(G[V \backslash\{v\}])\right),
$$

where $f_{l o w}, t(G)$ is the maximal flow that can be transferred from node $s$ to node $t$ in graph $G$-for an unweighted graph, this value is equal to the maximal number of edge-independent paths from $s$ to $t$ in a graph. We assume that flow $_{s, t}(V, E)=0$ if $s \notin V$ or $t \notin V$. The flow betweenness centrality satisfies Edge Balanced Contributions, as for each $s, t \in V$, the impact of an edge $e=\{v, u\}$ on the expression flow slt $_{s}(G)-$ flow $_{s, t}(G[V \backslash\{v\}])$ is non-zero if and only if edge $\{v, u\}$ increases flow low $_{s}(G)$; therefore, another edge, $e^{\prime}$, affects this impact if and only if both edges increase flow $_{s, t}(G)$. 
From Theorem 12, we know that there exists an induced game-theoretic centrality index that generates $c^{F}$. Let us identify such an induced index. Consider the index $\left[\left(r^{F}, \varphi^{\text {Shapley }}\right)\right]$, where $\varphi^{\text {Shapley }}$ is the Shapley value, and $r^{F}$ is defined as $f_{G}^{r^{F}}(S)=|V| \cdot f l o w_{s, t}(G[S])-$ $\sum_{v \in V} f_{l o w_{s, t}}(G[S \backslash\{v\}])$. For this $r^{F}$, it is possible to show that $\left[\left(r^{F}, \varphi^{\text {Shapley }}\right)\right]=c^{F}$.

With induced GTCs, the value of a coalition $S$ in a representation function depends both on the set of nodes, $S$, and the set of edges of the subgraph induced by $S$. However, $r^{F}$ in Example 4 depends solely on the edges of the subgraph induced by $S$. In the next section, we introduce our third class, namely Edge-Induced GTCs, characterized by such representation functions.

\section{Edge-Induced Game-Theoretic Centralities}

In this section, we define a subclass of induced GTCs which we call edge-induced gametheoretic centralities, or edge-induced GTCs.

Definition 4. (Edge-Induced GTC) A representation function, $r$, is edge-induced if for every two coalitions $S, S^{\prime} \subseteq V$ and every two graphs $G=(V, E), G^{\prime}=\left(V, E^{\prime}\right) \in \mathcal{G}^{V}$ such that $\{\{v, u\} \in E: v, u \in S\}=\left\{\{v, u\} \in E^{\prime}: v, u \in S^{\prime}\right\}$ it holds that $f_{G}^{r}(S)=f_{G^{\prime}}^{r}\left(S^{\prime}\right)$. A $G T C,(r, \varphi)$, is edge-induced if $r$ is edge-induced. The set of all edge-induced game-theoretic centralities is denoted by $\mathcal{E} \mathcal{I} \mathcal{G} \mathcal{C}$.

In words, a GTC is edge-induced if the value of a coalition $S$ in the representation function depends solely on the edges of the subgraph induced by $S$ in $G$. Thus, every edge-induced GTC is an induced GTC.

As we will show later on in this section, edge-induced GTCs are related to the notion of Balanced Contributions, proposed by Myerson (1980). Recall that contributions are balanced in a coalitional game if removing player $i$ from the game affects the payoff of player $j$ in the same way that removing player $j$ affects the payoff of player $i$. In the graph context, removing a node from the graph is interpreted as removing all its edges. More formally:

Balanced Contributions: For every graph, $G=(V, E)$, and every two nodes, $v, u \in V$, removing the edges of $v$ affects the centrality of $u$ in the same way that removing the edges of $u$ affects the centrality of $v$. More formally:

$$
c_{v}((V, E))-c_{v}\left(\left(V, E \backslash E_{u}\right)\right)=c_{u}((V, E))-c_{u}\left(\left(V, E \backslash E_{v}\right)\right) .
$$

Given a set of nodes, $V$, the class of all centrality indices that satisfy the Balanced Contributions property will be denoted by $\mathcal{C}_{B C}^{V}$, or just $\mathcal{C}_{B C}$ when $V$ is clear from the context.

The following lemma states that any centrality index in $\mathcal{C}_{B C}$ can be uniquely characterized by specifying (1) the centrality of every single-node in graph $(V, \emptyset)$; and $(2)$ the sum of node-centralities in every other graph in $\mathcal{G}^{V}$.

Lemma 13. For every function, $g: 2^{V} \times \mathcal{G}^{V} \rightarrow \mathbb{R}$, there exists at most one centrality index, $c \in \mathcal{C}_{B C}^{V}$, that satisfies: $\sum_{v \in V} c_{v}(G)=g(V, G)$ for every $G \in \mathcal{G}^{V} \backslash\{(V, \emptyset)\}$ and satisfies: $c_{v}((V, \emptyset))=g(\{v\},(V, \emptyset))$ for every $v \in V$. 
Proof. Let $c, c^{\prime} \in \mathcal{C}^{V}$ be two centrality indices such that the following holds:

$$
\begin{gathered}
\forall G \in \mathcal{G}^{V} \backslash\{(V, \emptyset)\}, \quad \sum_{v \in V} c_{v}(G)=g(V, G)=\sum_{v \in V} c_{v}^{\prime}(G), \\
\forall v \in V, \quad c_{v}((V, \emptyset))=g(\{v\},(V, \emptyset))=c_{v}^{\prime}((V, \emptyset)) .
\end{gathered}
$$

Then, to prove the correctness of the lemma, it suffices to prove that:

$$
\forall G \in \mathcal{G}^{V}, \forall v \in V, \quad c_{v}(G)=c_{v}^{\prime}(G) .
$$

This proof will proceed by contradiction. To this end, let $G=(V, E)$ be a graph with the minimum number of links such that $c(G) \neq c^{\prime}(G)$. Now, if $E=\emptyset$, we get a contradiction because of Equation (12). Next, let us assume that $E \neq \emptyset$, and let $v \in V$ be an arbitrary node in $G$. We will first show that Equation (13) holds when $v$ has no edges in $E$ (i.e., when $E_{v}=\emptyset$ ), and then show that it holds when $v$ has edges in $E$ (i.e., when $E_{v} \neq \emptyset$ ).

First, assume that $E_{v}=\emptyset$, and let $u_{1}, u_{2}, \ldots, u_{n-1}$ denote the nodes in $V \backslash\{v\}$. Then, based on the Balanced Contributions property of $c$, we have:

$$
\begin{aligned}
& c_{v}((V, E))-c_{v}\left(\left(V, E \backslash E_{u_{1}}\right)\right)=c_{u_{1}}((V, E))-c_{u_{1}}\left(\left(V, E \backslash E_{v}\right)\right)=0 ; \\
& c_{v}\left(\left(V, E \backslash E_{u_{1}}\right)\right)-c_{v}\left(\left(V, E \backslash E_{u_{1}} \backslash E_{u_{2}}\right)\right)=c_{u_{2}}\left(\left(V, E \backslash E_{u_{1}}\right)\right)-c_{u_{2}}\left(\left(V, E \backslash E_{u_{1}} \backslash E_{v}\right)\right)=0 ; \\
& c_{v}\left(\left(V, E \backslash E_{u_{1}} \backslash E_{u_{2}}\right)\right)-c_{v}\left(\left(V, E \backslash E_{u_{1}} \backslash E_{u_{2}} \backslash E_{u_{3}}\right)\right)=c_{u_{3}}\left(\left(V, E \backslash E_{u_{1}} \backslash E_{u_{2}}\right)\right)-c_{u_{3}}\left(\left(V, E \backslash E_{u_{1}} \backslash E_{u_{2}} \backslash E_{v}\right)\right)=0 ; \\
& c_{v}\left(\left(V, E \backslash \ldots \backslash E_{u_{n-3}}\right)\right)-c_{v}\left(\left(V, E \backslash \ldots \backslash E_{u_{n-2}}\right)\right)=c_{u_{n-2}}\left(\left(V, E \backslash \ldots \backslash E_{u_{n-3}}\right)\right)-c_{u_{n-2}}\left(\left(V, E \backslash \ldots \backslash E_{u_{n-3}} \backslash E_{v}\right)\right)=0 \\
& c_{v}\left(\left(V, E \backslash \ldots \backslash E_{u_{n-2}}\right)\right)-c_{v}\left(\left(V, E \backslash \ldots \backslash E_{u_{n-1}}\right)\right)=c_{u_{n-1}}\left(\left(V, E \backslash \ldots \backslash E_{u_{n-2}}\right)\right)-c_{u_{n-1}}\left(\left(V, E \backslash \ldots \backslash E_{u_{n-2}} \backslash E_{v}\right)\right)=0 .
\end{aligned}
$$

Based on this, we have:

$$
c_{v}((V, E))=c_{v}\left(\left(V, E \backslash E_{u_{1}}\right)\right)=c_{v}\left(\left(V, E \backslash E_{u_{1}} \backslash E_{u_{2}}\right)\right)=\ldots=c_{v}\left(\left(V, E \backslash E_{u_{1}} \ldots \backslash E_{u_{n-1}}\right)\right)=c_{v}((V, \emptyset)) .
$$

By performing the same calculations for $c^{\prime}$, we get:

$$
c_{v}^{\prime}((V, E))=c_{v}^{\prime}\left(\left(V, E \backslash E_{u_{1}}\right)\right)=c_{v}^{\prime}\left(\left(V, E \backslash E_{u_{1}} \backslash E_{u_{2}}\right)\right)=\ldots=c_{v}^{\prime}\left(\left(V, E \backslash E_{u_{1}} \ldots \backslash E_{u_{n-1}}\right)\right)=c_{v}^{\prime}((V, \emptyset)) .
$$

Based on Equations (12), (14), and (15), we find that Equation $(13) c_{v}((V, E))=c_{v}^{\prime}((V, E))$ holds.

Having proved that Equation (13) holds when $E_{v}=\emptyset$, it remains to show that it also holds when $E_{v} \neq \emptyset$. In this case, based on the Balanced Contributions property of $c$, the following holds for every $u \in V$ :

$$
c_{v}((V, E))-c_{v}\left(\left(V, E \backslash E_{u}\right)\right)=c_{u}((V, E))-c_{u}\left(\left(V, E \backslash E_{v}\right)\right) .
$$

Summing over all $u \in V$ (note that we consider also $u=v$ ), we get:

$$
|V| \cdot c_{v}((V, E))=\sum_{u \in V} c_{v}\left(\left(V, E \backslash E_{u}\right)\right)+\sum_{u \in V} c_{u}((V, E))-\sum_{u \in V} c_{u}\left(\left(V, E \backslash E_{v}\right)\right),
$$

which simplifies to

$$
\left(|V|-\mathcal{K}_{s}((V, E))\right) \cdot c_{v}((V, E))=\sum_{u \in V: E_{u} \neq \emptyset} c_{v}\left(\left(V, E \backslash E_{u}\right)\right)+\sum_{u \in V} c_{u}((V, E))-\sum_{u \in V} c_{u}\left(\left(V, E \backslash E_{v}\right)\right) .
$$


Recall that $\mathcal{K}_{s}(G)$ denotes the set of nodes that have no edges in $G$. Now, by performing the same calculations for $c^{\prime}$, and using the assumption that $G$ has a minimal number of links such that $c(G) \neq c^{\prime}(G)$, we have that for every $u \in V: E_{u} \neq \emptyset$ :

$$
c_{v}\left(\left(V, E \backslash E_{u}\right)\right)=c_{v}^{\prime}\left(\left(V, E \backslash E_{u}\right)\right) .
$$

and from Equation (11), we get:

$$
\left(|V|-\mathcal{K}_{s}((V, E))\right) \cdot c_{v}((V, E))=\left(|V|-\mathcal{K}_{s}((V, E))\right) \cdot c_{v}^{\prime}((V, E)) .
$$

Consequently, $c_{v}((V, E))=c_{v}^{\prime}((V, E))$ for every $v \in V$, which is a contradiction. This concludes the proof of Lemma 13.

Building upon the above lemma, the following theorem identifies a basis of the class $\mathcal{C}_{B C}$ (i.e., the class of all centrality indices that satisfy the Balanced Contributions property).

Theorem 14. $\mathcal{C}_{B C}$ is a vector space with the basis:

$$
\mathcal{U}^{B C}=\left\{c^{\langle\{v\}, \emptyset\rangle}: v \in V\right\} \cup\left\{c^{\left\langle U, E^{\dagger}\right\rangle}: E^{\dagger} \subseteq \mathcal{E}^{V}, E^{\dagger} \neq \emptyset, U=V \backslash K_{s}\left(\left(V, E^{\dagger}\right)\right)\right\} .
$$

Proof. If $c$ and $c^{\prime}$ satisfy Balanced Contributions, then $c+c^{\prime}$ and $k \cdot c$ satisfy Balanced Contributions, too, for every $k \in \mathbb{R}$. Thus, $\mathcal{C}_{B C}$ is a vector space. To prove that $\mathcal{U}_{B C}$ forms a basis of $\mathcal{C}_{B C}$ we show that $\mathcal{U}_{B C} \subseteq \mathcal{C}_{B C}$, that $\mathcal{U}_{B C}$ is linearly independent, and that $\left|\mathcal{U}_{B C}\right|$ is equal to the dimension of $\mathcal{C}_{B C}$.

Let us show that $\mathcal{U}_{B C} \subseteq \mathcal{C}_{B C}$, i.e., centralities from $\mathcal{U}_{B C}$ satisfy Balanced Contributions. To this end, we will consider different types of centralities from $\mathcal{U}^{B C}$ independently. Firstly, consider $c=c^{\langle\{v\}, \emptyset\rangle}$ for arbitrary $v \in V$. Since $c_{v}(G)$ does not depend on edges in $G$, i.e., $c_{v}\left(\left(V, E_{1}\right)\right)=c_{v}\left(\left(V, E_{2}\right)\right)$ for every $E_{1}, E_{2} \subseteq \mathcal{E}^{V}$, Balanced Contributions is trivially satisfied. Secondly, consider $c=c^{\left\langle U, E^{\dagger}\right\rangle}$ such that $U=V \backslash K_{s}\left(\left(V, E^{\dagger}\right)\right)$, and let $v, u \in V$ be two arbitrary nodes. If one of these two nodes, say $v$, does not have edges in $\left(V, E^{\dagger}\right)$ (i.e., $\left.v \in K_{s}\left(\left(V, E^{\dagger}\right)\right)\right)$, then $c_{v}((V, E))=0=c_{v}\left(\left(V, E \backslash E_{u}\right)\right)$, and from $E_{v}=\emptyset$ we get $c_{u}((V, E))=c_{u}\left(\left(V, E \backslash E_{v}\right)\right)$ which implies that the Balanced Contributions condition is satisfied. Assume otherwise, that $v$ and $u$ both have edges in $\left(V, E^{\dagger}\right)$. From the definition of unanimity centrality, we have that $c_{v}\left(\left(V, E \backslash E_{u}\right)\right)=0$, and $c_{u}\left(\left(V, E \backslash E_{v}\right)\right)=0$. Moreover, $c_{v}((V, E))=c_{u}((V, E))$ for every $E \subseteq \mathcal{E}^{V}$. Therefore:

$c_{v}((V, E))-c_{v}\left(\left(V, E \backslash E_{u}\right)\right)=c_{v}((V, E))-0=c_{u}((V, E))-0=c_{u}((V, E))-c_{u}\left(\left(V, E \backslash E_{v}\right)\right)$

for every $E \subseteq \mathcal{E}^{V}$ and thus Balanced Contributions is satisfied.

To show that $\mathcal{U}_{B C}$ is linearly independent, we use Lemma 2: if $c^{\left\langle U, E^{\dagger}\right\rangle}, c^{\left\langle U^{\prime}, E^{\dagger}\right\rangle} \in \mathcal{U}_{B C}$ $\left(U \neq U^{\prime}\right)$, then $U \cap U^{\prime}=\emptyset$ and the condition sufficient for linear independence is met.

Finally, we show that $\left|\mathcal{U}_{B C}\right|$ is equal to the dimension of $\mathcal{C}_{B C}$. Note that $\left|\mathcal{U}_{B C}\right|$ equals $\left|\mathcal{G}^{V}\right|-1+|V|$. Since centralities from $\mathcal{U}_{B C}$ are linearly independent, the dimension of the class $\mathcal{C}_{B C}$ is at least equal to $\left|\mathcal{U}_{B C}\right|$. But from Lemma 13 we know that it is at most equal to $\left|\mathcal{G}^{V}\right|-1+|V|$. Thus, the dimension of $\mathcal{C}_{B C}$ equals $\left|\mathcal{U}_{B C}\right|$. This concludes the proof of Theorem 14. 
Unlike in the previous sections, Balanced Contributions is too weak to characterize the class of Edge-Induced GTCs. To address this issue, we introduce a simple property called Zero-Empty.

Zero-Empty: For every node $v \in V$, the centrality of $v$ in an empty graph is zero, i.e., $c_{v}((V, \emptyset))=0$.

Given a set of nodes, $V$, the class of all centrality indices satisfying both Balanced Contributions and Zero-Empty will be denoted by $\mathcal{C}_{B C_{0}}^{V}$, or just $\mathcal{C}_{B C_{0}}$ when $V$ is clear from the context.

Theorem 14 states that every centrality satisfying Balance Contribution is a linear combination of centralities from $\mathcal{U}^{B C}$. Formally, there exists a collection of coefficients, $\alpha_{\left\langle U, E^{\dagger}\right\rangle}$ for every $c^{\left\langle U, E^{\dagger}\right\rangle} \in \mathcal{U}^{B C}$, that are not all equal to zero, such that:

$$
c=\sum_{v \in V} \alpha_{\langle\{v\}, \emptyset\rangle} c^{\langle\{v\}, \emptyset\rangle}+\sum_{E \subseteq \mathcal{E}^{V}: E \neq \emptyset, U=V \backslash K_{s}\left(\left(V, E^{\dagger}\right)\right)} \alpha_{\left\langle U, E^{\dagger}\right\rangle} c^{\left\langle U, E^{\dagger}\right\rangle} .
$$

From the definition of unanimity centrality we have that if $E^{\dagger} \neq 0$, then $c_{v}^{\left\langle U, E^{\dagger}\right\rangle}((V, \emptyset))=0$ for every $v \in V$. Therefore, $c_{v}((V, \emptyset))=\alpha_{\langle\{v\}, \emptyset\rangle}$. We get that a centrality, $c$, satisfies Zero-Empty if and only if $\alpha_{\langle\{v\}, \emptyset\rangle}=0$ for every $v \in V$. This is formalized in the following corollary.

Corollary 15. $\mathcal{C}_{B C_{0}}$ is a vector space with the basis:

$$
\mathcal{U}^{B C_{0}}=\left\{c^{\left\langle U, E^{\dagger}\right\rangle}: E^{\dagger} \subseteq \mathcal{E}^{V}, E^{\dagger} \neq \emptyset, U=V \backslash K_{s}\left(\left(V, E^{\dagger}\right)\right)\right\}
$$

To show that the class of edge-induced positive semivalue-based GTCs, denoted by $\mathcal{E} \mathcal{I} \mathcal{T} \mathcal{C}_{+}$, is characterized by the properties of Balanced Contributions and Zero-Empty, we will first show that every centrality from $\mathcal{E} \mathcal{I} \mathcal{G} \mathcal{C}_{+}$satisfies both properties.

Lemma 16. Every edge-induced game-theoretic centrality index in $\mathcal{G} \mathcal{T C} \mathcal{C}_{+}$satisfies Balanced Contributions and Zero-Empty.

Proof. Let $(r, \varphi)$ be an arbitrary GTC such that $r$ is edge-induced and $\varphi$ is a positive semivalue based on some $\beta$. First, we will prove that $[(r, \varphi)]$ satisfies Balanced Contributions. Then, we will consider Zero-Empty.

Let $G=(V, E)$ be an arbitrary graph and let $v, u \in V$ be two arbitrary distinct nodes. Considering the left-hand side of the condition of Balanced Contributions (Equation 10) and using Equation (1), we get:

$$
\begin{aligned}
& {[(r, \varphi)]_{v}((V, E))-[(r, \varphi)]_{v}\left(\left(V, E \backslash E_{u}\right)\right)} \\
& =\sum_{S \subseteq N, v \in S} \beta^{*}(|S|-1)\left(f_{(V, E)}^{r}(S)-f_{\left(V, E \backslash E_{u}\right)}^{r}(S)-f_{(V, E)}^{r}(S \backslash\{v\})+f_{\left(V, E \backslash E_{u}\right)}^{r}(S \backslash\{v\})\right) .
\end{aligned}
$$


Since $r$ is edge-induced, we know that if $u \notin S$, then $f_{(V, E)}^{r}(S)=f_{\left(V, E \backslash E_{u}\right)}^{r}(S)$. Therefore:

$$
\begin{aligned}
& {[(r, \varphi)]_{v}((V, E))-[(r, \varphi)]_{v}\left(\left(V, E \backslash E_{u}\right)\right) } \\
& =\sum_{S \subseteq N, v, u \in S} \beta^{*}(|S|-1)\left(f_{(V, E)}^{r}(S)-f_{\left(V, E \backslash E_{u}\right)}^{r}(S)-f_{(V, E)}^{r}(S \backslash\{v\})+f_{\left(V, E \backslash E_{u}\right)}^{r}(S \backslash\{v\})\right) \\
= & \sum_{S \subseteq N, v, u \in S} \beta^{*}(|S|-1)\left(f_{(V, E)}^{r}(S)-f_{(V, E)}^{r}(S \backslash\{u\})-f_{(V, E)}^{r}(S \backslash\{v\})+f_{(V, E)}^{r}(S \backslash\{v, u\})\right) .
\end{aligned}
$$

Here, we again used the fact that $r$ is edge-induced - since coalition $S$ has the same set of edges in $\left(V, E \backslash E_{u}\right)$ as coalition $S \backslash\{u\}$ in $(V, E)$, we have: $f_{\left(V, E \backslash E_{u}\right)}^{r}(S)=f_{(V, E)}^{r}(S \backslash\{u\})$. Analogously, we argue that $\left.\left.f_{\left(V, E \backslash E_{u}\right)}^{r}(S \backslash\{v\})\right)=f_{(V, E)}^{r}(S \backslash\{v, u\})\right)$. The expression obtained above is symmetric for $v$ and $u$. In other words, if we perform the same calculations for the right-hand side of the condition of Balanced Contributions, we will get the same result. Therefore:

$$
[(r, \varphi)]_{v}((V, E))-[(r, \varphi)]_{v}\left(\left(V, E \backslash E_{u}\right)\right)=[(r, \varphi)]_{u}((V, E))-[(r, \varphi)]_{u}\left(\left(V, E \backslash E_{v}\right)\right) .
$$

This concludes the proof that $(r, \varphi)$ satisfies Balanced Contributions.

Next, to see that $(r, \varphi)$ satisfies Zero-Empty, consider $f_{(V, \emptyset)}^{r}$. Since $r$ is edge-induced we have that $f_{(V, \emptyset)}^{r}(S)=f_{(V, \emptyset)}^{r}(\emptyset)=0$ for every $S \subseteq V$. Therefore, $f_{(V, \emptyset)}^{r}$ is a zero game and $[(r, \varphi)]_{v}((V, \emptyset))=0$ for every $v \in V$, which means that Zero-Empty is satisfied. This concludes the proof of Lemma 16.

Theorem 17. For every positive semivalue, $\varphi \in \mathcal{S V}_{+}$,

$$
\mathcal{C}_{B C_{0}}=\left[\mathcal{E} \mathcal{I} \mathcal{G} \mathcal{T} \mathcal{C}_{\varphi}\right]=[\mathcal{E} \mathcal{I} \mathcal{G} \mathcal{T C}+]
$$

Proof. Since $\mathcal{E I G T C}_{\varphi} \subseteq \mathcal{E} \mathcal{I} \mathcal{G} \mathcal{T} \mathcal{C}_{+}$, Lemma 16 implies that:

$$
\left[\mathcal{E} \mathcal{I} \mathcal{G} \mathcal{C}_{\varphi}\right] \subseteq\left[\mathcal{E} \mathcal{I} \mathcal{T} \mathcal{C}_{+}\right] \subseteq \mathcal{C}_{B C_{0}}
$$

It remains to prove that for every $\varphi \in \mathcal{S} \mathcal{V}_{+}$,

$$
\mathcal{C}_{B C_{0}} \subseteq\left[\mathcal{E} \mathcal{I} \mathcal{G} \mathcal{T} \mathcal{C}_{\varphi}\right]
$$

i.e., that every centrality, $c$, satisfying Balanced Contributions and Zero-Empty can be generated by $[(r, \varphi)]$ for some edge-induced representation function, $r$.

Based on Lemma 4, Theorem 14, and Corollary 15, we know that it suffices to prove that every unanimity centrality $c^{\left\langle U, E^{\dagger}\right\rangle} \in \mathcal{U}_{B C_{0}}$ can be generated by an edge-induced gametheoretic centrality from $\mathcal{G} \mathcal{T} \mathcal{C}_{+}$.

To this end, we use the representation function $r^{\left\langle U, E^{\dagger}\right\rangle}$ from the proof of Theorem 12, defined as follows:

$$
f_{(V, E)}^{r^{\left\langle U, E^{\dagger}\right\rangle}}(S)= \begin{cases}\left(\sum_{k=|U|}^{|V|}\left(\begin{array}{c}
|V|-|U| \\
k-|U|
\end{array}\right) \beta^{*}(k-1)\right)^{-1} & \text { if } U \subseteq S, E^{\dagger} \subseteq E, \\
0 & \text { otherwise. }\end{cases}
$$


We already showed in the proof of Theorem 12 that $\left[\left(r^{\left\langle U, E^{\dagger}\right\rangle}, \varphi\right)\right]=c^{\left\langle U, E^{\dagger}\right\rangle}$. Since for every unanimity game $c^{\left\langle U, E^{\dagger}\right\rangle}$ from the class $\mathcal{U}_{B C_{0}}$ we have $U=V \backslash K_{s}\left(\left(V, E^{\dagger}\right)\right)$, then to generate every unanimity centrality from $\mathcal{U}_{B C_{0}}$ we use the following GTCs:

$$
\left\{\left(r^{\left\langle U, E^{\dagger}\right\rangle}, \varphi\right): E^{\dagger} \subseteq \mathcal{E}^{V}, E^{\dagger} \neq \emptyset, U=V \backslash K_{s}\left(\left(V, E^{\dagger}\right)\right)\right\}
$$

It remains to prove that, for every $E^{\dagger} \subseteq \mathcal{E}^{V}, E^{\dagger} \neq \emptyset$ and $U=V \backslash K_{s}\left(\left(V, E^{\dagger}\right)\right)$, the function $r^{\left\langle U, E^{\dagger}\right\rangle}$ is edge-induced. From the definition we have that $f_{G}^{r^{\left\langle U, E^{\dagger}\right\rangle}}(S)$ for $U=V \backslash K_{s}\left(\left(V, E^{\dagger}\right)\right)$ does not equal zero if and only if the graph $G$ contains all edges from $E^{\dagger}$ and $S$ contains all nodes with edges in $E^{\dagger}$. Moreover, if the value of a coalition is not equal to zero, then it depends solely on the size of $|U|$. Therefore, for every two graphs $G, G^{\prime} \in \mathcal{G}^{V}$, and every two coalitions $S, S^{\prime} \subseteq V$ such that $G[S]$ contains exactly the same edges as $G^{\prime}\left[S^{\prime}\right]$, the value is equal. Thus, $r^{\left\langle U, E^{\dagger}\right\rangle}$ is edge-induced. This concludes the proof of Theorem 17.

\section{Discussion \& Conclusion}

We proposed an axiomatic characterization of game-theoretic centralities. Our results are summarized in Figure 4. We showed that, while all centralities can be obtained by the gametheoretic approach, some natural classes of game-theoretic centralities are characterized by Fairness and its strengthenings - Edge Balanced Contributions and Balanced Contributions.

Our work has a number of implications.

- Where to use game-theoretic centralities: Our results suggest that the game-theoretic approach is a good choice when the nodes are assessed based on some property that adheres to Fairness. A good example of such a property is "connectivity" (Michalak, Rahwan, Szczepański, Skibski, Narayanam, Wooldridge, \& Jennings, 2013; Skibski et al., 2016). In measuring connectivity, Fairness seems to be a reasonable assumption, as both nodes connected by an edge should benefit the same from the fact this edge increases connectivity, i.e., it is used to keep the graph connected. On the other hand, in many settings Fairness may not be intuitive. In particular, in social networks, a link between two people may be much more profitable for the one with a lower social standing.

- Designing new, fair centrality measures: We proved that every centrality that satisfies Fairness can be obtained from a separable game-theoretic centrality (Theorem 8). This result suggests an appealing way to define fair centrality measures that rely on assessing not nodes, but the subnetworks of the network - as in the representation function. For example, let us consider in more detail the aforementioned notion of connectivity. We already argued that it should satisfy Fairness. Now, we can assign to each group a value that reflect how well-connected is the group. To this end, we can assign value 0 to a group of nodes that form an independent set, value 1 to a group with only two nodes connected to each other, and so on and so forth. More formally, we can define a representation function as follows: $f_{G}^{r}(S)=|S|-|K(G[S])|$. In this way, using the Shapley value as the solution concept, we obtain the Attachment centrality (divided by 2), discussed in the related work section. We believe the same method can be used to capture other properties of nodes. 


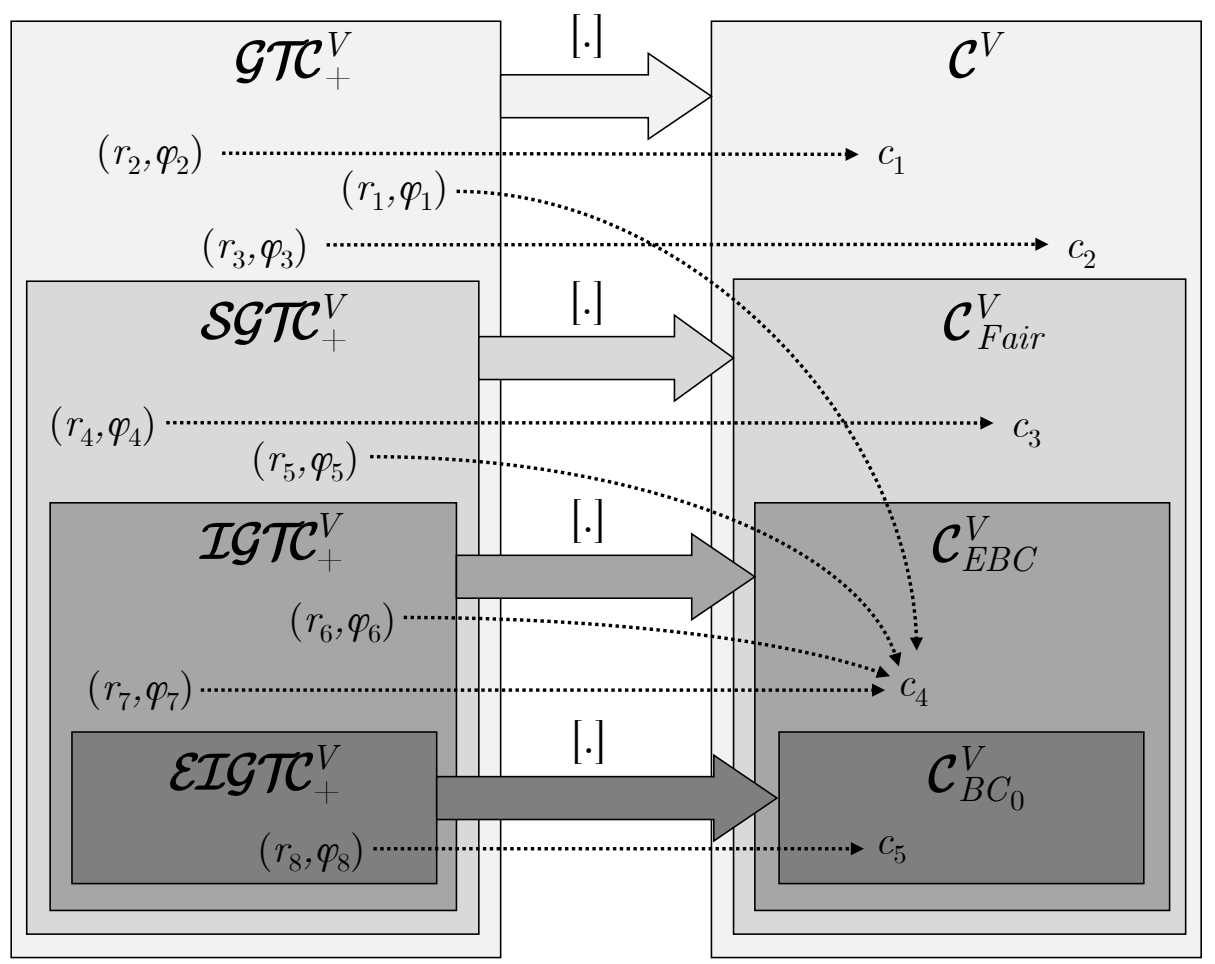

Figure 4: An illustration summarizing our results. Every small (dotted) arrow connects a game-theoretic centrality, $(r, \varphi)$, to the corresponding centrality $c=[(r, \varphi)]$ (note that all the game-theoretic centralities and centralities from this example, such as $\left(r_{3}, \varphi_{3}\right)$ and $c_{2}$, are just for illustrative purposes and do not correspond to any specific measures from the literature). Furthermore, every large arrow connects some subclass of GTCs, $\mathcal{I}$, to $[\mathcal{I}]$ - the class of centrality indices generated by $\mathcal{I}$. As we have shown in Theorem $1,\left[\mathcal{G} \mathcal{T} \mathcal{C}_{+}\right]$encompasses all centralities, i.e., for every centrality, $c$, there is an incoming arrow from some game-theoretic centrality $(r, \varphi) \in \mathcal{G} \mathcal{T} \mathcal{C}_{+}$. In Theorem 8, we proved that all arrows from Separable GTCs go into centralities that satisfy Fairness, and that for every centrality satisfying Fairness there exists an incoming arrow from some Separable GTC. In Theorem 12, we showed that all arrows from Induced GTCs go into centralities that satisfy Edge Balanced Contributions, and that for every centrality satisfying Edge Balanced Contributions there exists an incoming arrow from some induced game-theoretic centrality. In Theorem 17, we proved that all arrows from EdgeInduced GTCs go into centralities that satisfy Balanced Contributions and ZeroEmpty, and that for every centrality satisfying Balanced Contributions and ZeroEmpty there exists an incoming arrow from some edge-induced game-theoretic centrality. Note that it is possible that several game-theoretic centralities generate the same centrality (e.g., $\left.\left[\left(r_{5}, \varphi_{5}\right)\right]=\left[\left(r_{6}, \varphi_{6}\right)\right]\right)$ or that a non-separable gametheoretic centrality generates a centrality satisfying Fairness or Edge Balanced Contributions (e.g., $\left.\left[\left(r_{1}, \varphi_{1}\right)\right]=c_{4}\right)$. 
- Theoretical foundations of centrality measures: Finally, it is worth to highlight the fact that some of the theoretical foundations introduced in this paper are applicable not only to game-theoretic centrality measures but, more generally, to any centrality measure. In particular, we defined a class of unanimity centralities (Definition 1) and, building upon this definition, we specified the basis of the class of all centralities, and of the centralities that satisfy Fairness and its extensions (Theorems 3, 6, 10, and 14). To the best of our knowledge, the techniques proposed by us are new and they can be used to further axiomatic analysis of centrality measures. In fact, the early versions of these results were already used by us in the work on axiomatic foundations of the Attachment centrality's extension to weighted graphs (Sosnowska \& Skibski, 2017).

In our future work, we plan to further study the class of game-theoretic centralities for directed graphs. Also, we are interested in studying a link to the large class of vitality indices, where the centrality of a node is a difference between the value of a network with and without a node in question (Brandes \& Erlebach, 2005).

\section{Acknowledgements}

This article is an extended version of a paper originally presented at AAAI-17 (Skibski, Michalak, \& Rahwan, 2017) with a number of new technical results. Specifically, all the results regarding the class of edge-induced game-theoretic centralities are new (Section 7). Furthermore, we added proofs of Lemma 2, 4, 5, 7, 9, 11 and full proofs (instead of sketches) of Theorem 6, 8, 10, 12 (Sections 4-6). Finally, we extended the related work section (Section 2) and added the discussion of the implications of our work (Section 8).

Oskar Skibski was supported by the Foundation for Polish Science within the Homing programme financed from the Smart Growth Operational Programme (Project title: "Centrality Measures: from Theory to Applications"). Tomasz Michalak and Talal Rahwan were supported by the Polish National Science Center grant DEC-2013/09/D/ST6/03920. Tomasz Michalak was also supported by the European Research Council under Advanced Grant 291528 ("RACE") for the earlier version of this work.

\section{Appendix A. Summary of Notation}

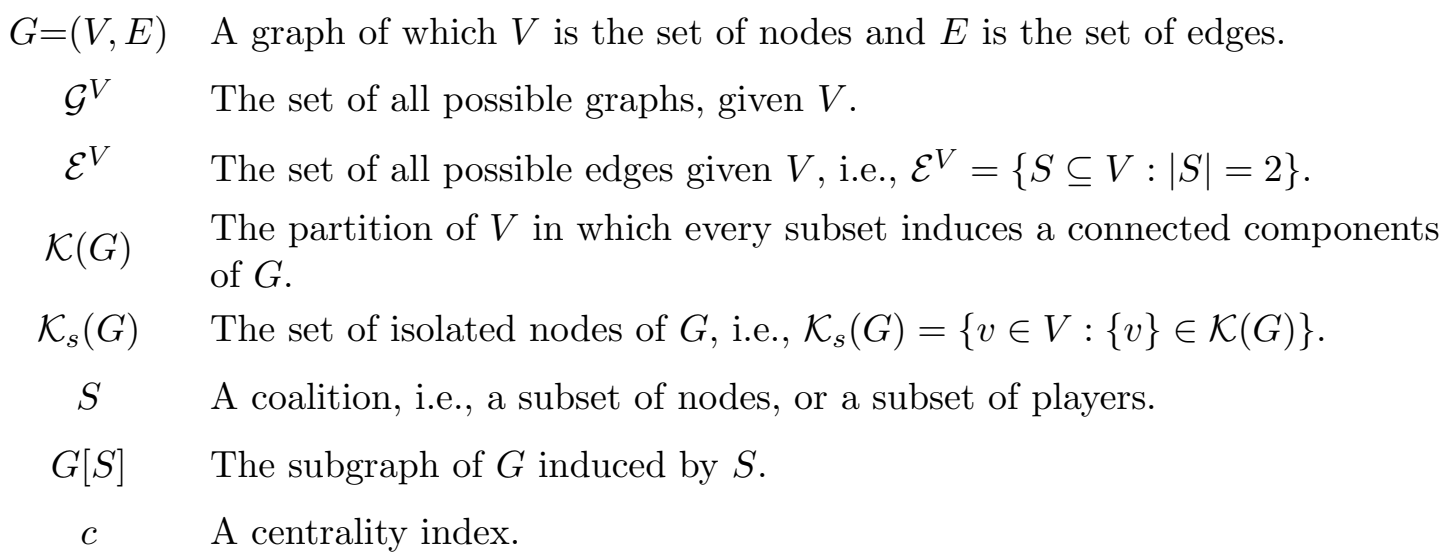


$c_{v}(G) \quad$ The centrality of $v$ in $G$ according to $c$.

$\mathcal{C}^{V} \quad$ The set of all centrality indices, given $V$.

$\mathcal{C}_{\text {Fair }}^{V} \quad$ The set of all centrality indices satisfying Fairness, given $V$.

$\mathcal{C}_{E B C}^{V} \quad$ The set of all centrality indices satisfying Edge Balanced Contributions, given $V$.

$\mathcal{C}_{B C}^{V} \quad$ The set of all centrality indices satisfying Balanced Contributions, given $V$.

$\mathcal{C}_{B C_{0}}^{V} \quad$ The set of all centrality indices satisfying Balanced Contributions and ZeroEmpty, given $V$.

$(N, f) \quad$ A game of which $N$ is the set of players and $f$ is the characteristic function. $\mathcal{F}^{N} \quad$ The set of all possible games, given $N$.

$\varphi \quad$ A solution concept.

$\varphi_{v}(f) \quad$ The payoff of $v$ in game $(N, f)$ according to $\varphi$.

$\Phi^{N} \quad$ The set of all solution concepts, given $N$.

$\beta \quad$ A function $\beta:\{0, \ldots,|N|-1\} \rightarrow[0,1]$ such that $\sum_{k=0}^{|N|-1} \beta(k)=1$.

$\varphi^{\beta} \quad$ The semivalue defined by $\beta$.

$\beta^{*}(k) \quad$ A shorthand notation for $\beta(k) /\left(\begin{array}{c}|N|-1 \\ k\end{array}\right)$.

$\mathcal{S} \mathcal{V}^{N} \quad$ The set of all semivalues, given $N$.

$\mathcal{S} \mathcal{V}_{+}^{N} \quad$ The set of all positive semivalues, given $N$.

A representation function; it takes a graph, $G=(V, E)$, and returns a game $\left(V, f_{G}^{r}\right)$.

$\mathcal{R}^{V} \quad$ The set of representation functions, given $V$.

$(r, \varphi) \quad$ A game-theoretic centrality index.

$[(r, \varphi)]$ The centrality generated by $(r, \varphi)$, i.e., for every node, $v \in V$, we have: $[(r, \varphi)]_{v}(G)=\varphi_{v}\left(f_{G}\right)$.

$\mathcal{G T C}^{V} \quad$ The set of game-theoretic centrality indices, given $V$.

$\mathcal{G T C}_{+}^{V} \quad$ The set of game-theoretic centrality indices based on positive semivalues, given $V$.

$\mathcal{G T C}_{\varphi}^{V} \quad$ The set of game-theoretic centrality indices based on $\varphi$, given $V$.

$\mathcal{S} \mathcal{G} \mathcal{C C}^{V} \quad$ The set of separable game-theoretic centrality indices, given $V$.

$\mathcal{I} \mathcal{G} \mathcal{C}^{V} \quad$ The set of induced game-theoretic centrality indices, given $V$.

$\mathcal{E I G T C}^{V}$ The set of edge-induced game-theoretic centrality indices, given $V$.

$c^{\left\langle U, E^{\dagger}\right\rangle} \quad$ A unanimity centrality index.

$\mathcal{U}^{V} \quad$ The set of all unanimity centrality indices, given $V$. 


\section{References}

Amer, R., \& Giménez, J. M. (2004). A connectivity game for graphs. Mathematical Methods of Operations Research, 60(3), 453-470.

Banzhaf III, J. F. (1965). Weighted voting doesn't work: A game theoretic approach. Rutgers Law Review, 19 (317), 343.

Bianzino, A. P., Chaudet, C., Rossi, D., Rougier, J.-L., \& Moretti, S. (2011). The greengame: Striking a balance between qos and energy saving. In Teletraffic Congress (ITC), 2011 23rd International, pp. 262-269.

Boldi, P., \& Vigna, S. (2014). Axioms for centrality. Internet Mathematics, 10(3-4), 222262.

Borgatti, S. P. (2005). Centrality and network flow. Social networks, 27(1), 55-71.

Borgatti, S. P., \& Everett, M. G. (2006). A graph-theoretic perspective on centrality. Social Networks, 28(4), 466-484.

Brandes, U. (2014). Social network algorithmics. In Proceeding of 25th International Symposium on Algorithms and Computation (ISAAC), pp. XV-XVI.

Brandes, U., \& Erlebach, T. (2005). Network analysis: Methodological foundations. SpringerVerlag.

Brânzei, S., \& Larson, K. (2009). Coalitional affinity games and the stability gap. In Proceedings of the 21st International Joint Conference on Artificial Intelligence (IJCAI), pp. 1319-1320.

Brânzei, S., \& Larson, K. (2011). Social distance games. In Proceedings of the 22nd International Joint Conference on Artificial Intelligence (IJCAI), pp. 1281-1282.

Chalkiadakis, G., Elkind, E., \& Wooldridge, M. (2011). Computational aspects of cooperative game theory. Synthesis Lectures on Artificial Intelligence and Machine Learning, 5(6), 1-168.

del Pozo, M., Manuel, C., González-Arangüena, E., \& Owen, G. (2011). Centrality in directed social networks. a game theoretic approach. Social Networks, 33(3), 191200.

Deng, X., \& Papadimitriou, C. H. (1994). On the complexity of cooperative solution concepts. Mathematics of Operations Research, 19(2), 257-266.

Dubey, P., Neyman, A., \& Weber, R. J. (1981). Value theory without efficiency. Mathematics of Operations Research, 6(1), 122-128.

Elkind, E., \& Wooldridge, M. (2009). Hedonic coalition nets. In Proceedings of the 8th International Conference on Autonomous Agents and Multiagent Systems (AAMAS), pp. 417-424.

Freeman, L. C., Borgatti, S. P., \& White, D. R. (1991). Centrality in valued graphs: A measure of betweenness based on network flow. Social Networks, 13(2), 141-154.

Gomez, D., González-Arangüena, E., Manuel, C., Owen, G., del Pozo, M., \& Tejada, J. (2003). Centrality and power in social networks: A game theoretic approach. Mathematical Social Sciences, 46(1), 27-54. 
Ieong, S., \& Shoham, Y. (2005). Marginal contribution nets: A compact representation scheme for coalitional games. In Proceedings of the 6th ACM Conference on Electronic Commerce (ACM-EC), pp. 193-202.

Koschützki, D., Lehmann, K. A., Peeters, L., Richter, S., Tenfelde-Podehl, D., \& Zlotowski, O. (2005). Centrality indices. In Network Analysis, Vol. 3418 of Lecture Notes in Computer Science, pp. 16-61. Springer.

Kötter, R., Reid, A. T., Krumnack, A., Wanke, E., \& Sporns, O. (2007). Shapley ratings in brain networks. Frontiers in neuroinformatics, 1, 2.

Lindelauf, R., Hamers, H., \& Husslage, B. (2013). Cooperative game theoretic centrality analysis of terrorist networks: The cases of Jemaah Islamiyah and Al Qaeda. European Journal of Operational Research, 229(1), 230-238.

Maschler, M., Solan, E., \& Zamir, S. (2013). Game Theory. Cambridge University Press.

Michalak, T. P., Aadithya, K. V., Szczepański, P. L., Ravindran, B., \& Jennings, N. R. (2013). Efficient computation of the Shapley value for game-theoretic network centrality. Journal of Artificial Intelligence Research, 46, 607-650.

Michalak, T. P., Rahwan, T., Skibski, O., \& Wooldridge, M. (2015). Defeating terrorist networks with game theory. IEEE Intelligent Systems, 30(1), 53-61.

Michalak, T. P., Rahwan, T., Szczepański, P. L., Skibski, O., Narayanam, R., Wooldridge, M., \& Jennings, N. R. (2013). Computational analysis of connectivity games with applications to the investigation of terrorist networks. In Proceedings of the 23rd International Joint Conference on Artificial Intelligence (IJCAI), pp. 293-301.

Moretti, S., Fragnelli, V., Patrone, F., \& Bonassi, S. (2010). Using coalitional games on biological networks to measure centrality and power of genes. Bioinformatics, 26(21), $2721-2730$.

Myerson, R. B. (1977). Graphs and cooperation in games. Mathematical Methods of Operations Research, 2(3), 225-229.

Myerson, R. B. (1980). Conference structures and fair allocation rules. International Journal of Game Theory, 9, 169-82.

Narayanam, R., \& Narahari, Y. (2011). A shapley value-based approach to discover influential nodes in social networks. Automation Science and Engineering, IEEE Transactions on, 8(1), 130-147.

Narayanam, R., Skibski, O., Lamba, H., \& Michalak, T. P. (2014). A Shapley value-based approach to determine gatekeepers in social networks with applications. In Proceedings of the 21st European Conference on Artificial Intelligence (ECAI), pp. 651-656.

Nieminen, J. (1974). On the centrality in a graph. Scandinavian journal of psychology, $15(1), 332-336$.

Sabidussi, G. (1966). The centrality index of a graph. Psychometrika, 31(4), 581-603.

Schoch, D., \& Brandes, U. (2015). Stars, neighborhood inclusion, and network centrality. In SIAM Workshop on Network Science. 
Shapley, L. S. (1953). A value for n-person games. In Kuhn, H., \& Tucker, A. (Eds.), Contributions to the Theory of Games, volume II, Vol. II, pp. 307-317. Princeton University Press.

Skibski, O., Michalak, T. P., \& Rahwan, T. (2017). Axiomatic characterization of gametheoretic network centralities. In Proceedings of the 31st AAAI Conference on Artificial Intelligence (AAAI), pp. 698-705.

Skibski, O., Michalak, T. P., Rahwan, T., \& Wooldridge, M. (2014). Algorithms for the Shapley and Myerson values in graph-restricted games. In Proceedings of the 13th International Conference on Autonomous Agents and Multiagent Systems (AAMAS), pp. 197-204.

Skibski, O., Rahwan, T., Michalak, T. P., \& Yokoo, M. (2016). Attachment centrality: An axiomatic approach to connectivity in networks. In Proceedings of the 15th International Conference on Autonomous Agents and Multiagent Systems (AAMAS), pp. $168-176$.

Sosnowska, J., \& Skibski, O. (2017). Attachment centrality for weighted graphs. In Proceedings of the 26th International Joint Conference on Artificial Intelligence (IJCAI), pp. 416-422.

Suri, N. R., \& Narahari, Y. (2008). Determining the top-k nodes in social networks using the Shapley value. In Proceedings of the 7th International Conference on Autonomous Agents and Multiagent Systems (AAMAS), pp. 1509-1512.

Szczepański, P. L., Michalak, T. P., \& Rahwan, T. (2016). Efficient algorithms for gametheoretic betweenness centrality. Artificial Intelligence, 231, 39-63.

Winter, E. (2002). The Shapley value. Handbook of game theory with economic applications, 3, 2025-2054. 